\title{
Kinetic Theory of Nonlinear Viscous Flow in Two and Three Dimensions
}

\author{
M. H. Ernst, ${ }^{1}$ B. Cichocki, ${ }^{1,2}$ J. R. Dorfman, ${ }^{3}$ J. Sharma, ${ }^{3}$ \\ and H. van Beijeren ${ }^{4}$
}

Received August 2, 1977

\begin{abstract}
On the basis of a nonlinear kinetic equation for a moderately dense system of hard spheres and disks it is shown that shear and normal stresses in a steady-state, uniform shear flow contain singular contributions of the form $|X|^{3 / 2}$ for hard spheres, or $|X| \log |X|$ for hard disks. Here $X$ is proportional to the velocity gradient in the shear flow. The origin of these terms is closely related to the hydrodynamic tails $t^{-d / 2}$ in the current-current correlation functions. These results also imply that a nonlinear shear viscosity exists in two-dimensional systems. An extensive discussion is given on the range of $X$ values where the present theory can be applied, and numerical estimates of the effects are given for typical circumstances in laboratory and computer experiments.
\end{abstract}

KEY WORDS: Kinetic theory; nonlinear transport properties; uniform steady-state shear flow; non-Newtonian fluid properties; hydrodynamic long-time tails.

\section{INTRODUCTION}

During the past several years, evidence has been accumulating that fundamental difficulties are encountered in the derivation of hydrodynamic equations for two-dimensional gases whose particles interact with shortranged repulsive forces. ${ }^{(1-5)}$ This evidence comes from both computer and theoretical studies of time correlation functions for two-dimensional systems

Supported by National Science Foundation grant No. CHE-73-08856 (to HvB, JRD, and JS) and the Center for Theoretical Physics of the Univ. of Md. (to HvB).

${ }^{1}$ Instituut voor Theoretische Fysica der Rijksuniversiteit Utrecht, The Netherlands.

${ }^{2}$ On leave from Institute of Theoretical Physics, Warsaw University, Warsaw, Poland.

${ }^{3}$ Institute for Physical Science and Technology, and Department of Physics and Astronomy, University of Maryland, College Park, Maryland.

${ }^{4}$ Institut für Theoretische Physik, RWTH, Aachen, West Germany. 
composed of hard-disk molecules. The motivation for these studies is based on the fact that if linearized hydrodynamic equations apply to two-dimensional (or higher) systems that are sufficiently close to equilibrium, then the transport coefficients associated with these hydrodynamic equations can be expressed as time integrals of time correlation functions, ${ }^{(6)}$ through relations of the form

$$
L_{\mu}=\int_{0}^{\infty} d t\left\langle J_{\mu}(t) J_{\mu}(0)\right\rangle_{\mathrm{eq}}
$$

where $L_{\mu}$ is a transport coefficient, such as the coefficient of self-diffusion $D$, shear viscosity $\eta$, or thermal conductivity $\lambda$. The microscopic current $J_{\mu}$ can be expressed in terms of the positions and momenta of the particles of the system and the potential energy between the particles. $J_{\mu}(0)$ is the value of $J_{\mu}$ at some initial time and $J_{u}(t)$ is that at a time $t$ later. The angular brackets denote an average over an equilibrium ensemble. It was discovered, first by computer studies on the velocity autocorrelation function, which is related to the coefficient of self-diffusion, and later by theoretical studies on this and the correlation functions related to $\eta$ and $\lambda$, that for moderately dense gases there is a range of times $t$ over which the current correlation functions behave like

$$
\left\langle J_{\mu}(0) J_{\mu}(t)\right\rangle_{\mathrm{eq}}=a_{\mu}\left(t_{0} / t\right)^{d / 2}
$$

Here, $a_{\mu}$ is a constant, depending on $\mu$, and $t_{0}$ is the mean free time between collisions for a particle in a gas. For the presently available computer studies on the velocity autocorrelation function the $t^{-d / 2}$ behavior persists over the time range $10 t_{0} \leqslant t \leqslant 200 t_{0}$, the upper limit being determined by the size of the system studied. Although the other time correlation functions have not been studied as extensively, both the computer work that has been done and theoretical studies suggest that similar results also hold for the correlation functions related to $\eta$ and $\lambda$.

If the $t^{-1}$ decay of the time correlation functions for two-dimensional systems would persist for arbitrarily long times, then Eq. (1) would have as a consequence that the associated transport coefficients are infinite. For threedimensional systems, the $t^{-3 / 2}$ decay is sufficiently fast that the transport coefficients associated with the linear hydrodynamic equations are all finite, but divergence difficulties occur when one considers the transport coefficients associated with both the linear and nonlinear Burnett and higher order hydrodynamic equations. ${ }^{(7-14)}$

In spite of the fact that neither computer nor theoretical studies have shown that the transport coefficients do in fact diverge, the fact that the current correlation functions decay so slowly for long times provides a sufficiently strong motivation for a reexamination of the derivation of the linearized 
hydrodynamic equations from first principles. This should reveal whether the apparent divergence of the associated transport coefficient implies that nonlinear and nonlocal effects neglected in their derivation should be taken into account.

In this paper we use the kinetic theory of gases to derive the hydrodynamic equations that describe steady-state shear flow and we consider both twoand three-dimensional systems. By generalizing a method originally used by Ernst and Dorfman ${ }^{(7)}$ for the derivation of linear hydrodynamic equations, we have been able to show that for two-dimensional systems the appropriate hydrodynamic equations are necessarily nonlinear. We have derived the nonlinear hydrodynamic equations that describe shear flow and we show that the associated transport coefficients are all finite. The three-dimensional version of the theory provides a more fundamental derivation of the results of Kawasaki and Gunton ${ }^{(14)}$ and Yamada and Kawasaki, ${ }^{(15)}$ who obtained nonlinear effects in shear flow from a generalization of the mode coupling theory. In the paper we do not consider more general time-dependent problems involving heat flow, since the derivation of the general hydrodynamic equations seems to be considerably more complex than that for steady shear flow and work on the general case is still in progress.

Here we will consider a steady shear flow when the local velocity $\mathbf{u}(\mathbf{r})$ is in the $\hat{\mathbf{y}}$ direction and its value depends on the $x$ coordinate, i.e., $\mathbf{u}(\mathbf{r})=\hat{\mathbf{y}} f(x)$, where $\hat{\mathbf{y}}$ is a unit vector in the $\hat{\mathbf{y}}$ direction and $f(x)$ is a function of $x$ alone. We consider the simplest case, where $f(x)$ is linear in $x$, so that

$$
u_{\alpha}(\mathbf{r})=\delta_{\alpha y}\left(u_{0}+x X\right)
$$

The quantity $X$ characterizes the size of the velocity gradient, and $\alpha, \beta, \gamma$ denote Cartesian components $x, y, z$. We will compute elements of the pressure tensor, such as $P_{x y}$, and the ratio $P_{x y} \mid X$. If the linear hydrodynamic equations are valid, then

$$
\lim _{X \rightarrow 0} P_{x y} / X=-\eta
$$

where $\eta$ is the coefficient of shear viscosity. In the time correlation function method it is assumed that the limit (4) exists.

In order to provide a motivation for the work carried out in this paper, we mention that the $t^{-d / 2}$ behavior of the time correlation function is associated with certain dynamical processes taking place in the gas, the so-called ring events. ${ }^{(3,7)}$ These events consist of a sequence of $l$ binary collisions taking place among $l$ particles in the gas, for $l \geqslant 3$. It has been shown that these events collectively lead to contributions to the time correlation functions that contain integrals over wave numbers $k$ of the form

$$
\int_{0}^{k_{0}} d k k^{d-1} \exp \left(-a k^{2} t\right)
$$


where $d$ is the number of dimensions, $k_{0} \sim n \sigma^{d-1}$ is a cutoff wave number, which for dilute and moderately dense gases is of the order of the inverse mean free path, and $a$ is a quantity of the order of $v_{0} / n \sigma^{d-1}$, where $v_{0}$ is the thermal velocity of a particle in the gas. By carrying out the $k$ integral we obtain the $t^{-d / 2}$ behavior for long time $t \gg t_{0}$. In this paper we include effects that are neglected in the derivation of the linearized hydrodynamic equations and are due to the presence of velocity gradients in the shear flow. We show that the ratio $P_{x y} / X$ has contributions of the form

$$
P_{x y} / X \simeq \int_{0}^{\infty} d t \int_{0}^{k_{0}} d k k^{d-1} \exp \left(-a k^{2} t-b|X| t\right)
$$

For two-dimensional systems this gives

$$
P_{x y} \simeq X \log |X|
$$

Thus the ring events lead to a finite value of the pressure tensor if nonlinear effects are taken into account. For three-dimensional systems, similar nonlinear effects lead to

$$
P_{x y} \simeq X\left(-\eta+c|X|^{1 / 2}\right)
$$

as predicted earlier by Kawasaki, Gunton, and Yamada. ${ }^{5}$

The plan of the paper is as follows. In Section 2 we start from the BBGKY hierarchy equations and derive equations for the single and pair distribution functions that take into account the ring events and nonlinear effects of the velocity field on the contribution to the pressure tensor. In Section 3 we compute the contribution of the ring events to the pressure tensor and show that the effects described in Eq. (5) come from the so-called hydrodynamic mode contributions. We then verify Eqs. (6) and (7), and give in Section 4 the precise numerical factors that are of interest for all elements of $P_{\alpha \beta}$. We conclude in Section 5 with a discussion of our results and a comparison of our results with those of other authors.

\section{NONLINEAR TRANSPORT EQUATIONS}

We consider a classical $d$-dimensional system consisting of $N$ particles contained in a volume $V$. We take the particles to be $d$-dimensional hard spheres, each of mass $m$ and diameter $\sigma$. We will discuss the nonequilibrium one-particle, pair,..., distribution functions $f(1, t), f(12, t), \ldots$, respectively,

${ }^{5}$ While this work was in progress, we received the $\mathrm{PhD}$ dissertation of Onuki, ${ }^{(16)}$ which contains results similar, and in some cases identical, to those presented here. Unfortunately, Onuki does not indicate how he arrives at his results, so that we cannot compare our method with his, other than the fact that we both start from the BBGKY hierarchy. 
where $x_{i}$ denptes the phase $x_{i}=\left(\mathbf{r}_{i}, \mathbf{v}_{i}\right)$ of particle $i$, and $\mathbf{r}_{i}$ and $\mathbf{v}_{i}$ are the position and velocity of the $i$ th particle.

First, we derive a nonlinear kinetic equation from the BBGKY hierarchy, and we apply the Chapman-Enskog method ${ }^{(17)}$ to obtain transport equations and expressions for the fluxes. For hard-sphere systems the distribution functions satisfy the following BBGKY hierarchy ${ }^{(18)}$ :

$$
\begin{gathered}
\left(\partial_{t}+\mathbf{v}_{1} \cdot \frac{\partial}{\partial \mathbf{r}_{1}}\right) f(1)=\int d x_{2} T(12) f(12) \\
{\left[\partial_{t}+\mathbf{v}_{1} \cdot \frac{\partial}{\partial \mathbf{r}_{1}}+\mathbf{v}_{2} \cdot \frac{\partial}{\partial \mathbf{r}_{2}}-T(12)\right] f(12)=\int d x_{3}\left(1+P_{12}\right) T(13) f(123)}
\end{gathered}
$$

The operator $P_{i j}$ interchanges the particle labels $(i j)$, and the binary collision operators ${ }^{(18)}$ used here are point $T$-operators, which neglect the difference in position of colliding particles (a low-density approximation)

$$
T(12)=\delta\left(\mathbf{r}_{12}\right) T_{0}(12)=\delta\left(\mathbf{r}_{12}\right) \sigma^{d-1} \int_{\mathbf{v}_{12} \cdot \hat{\jmath}>0} d \hat{\sigma}\left|\mathbf{v}_{12} \cdot \hat{\boldsymbol{\sigma}}\right|\left[b_{\sigma}(12)-1\right]
$$

The unit vector $\hat{\sigma}$ denotes the direction of the line of centers of the colliding pair. Here, $b_{\sigma}(12)$ changes $\mathbf{v}_{1}$ and $\mathbf{v}_{2}$ into restituting velocities $\mathbf{v}_{1}{ }^{\prime}$ and $\mathbf{v}_{2}{ }^{\prime}$. We further use the notation $\mathbf{v}_{12}=\mathbf{v}_{1}-\mathbf{v}_{2}$ and $\mathbf{r}_{12}=\mathbf{r}_{1}-\mathbf{r}_{2}$. Next, we introduce the cluster functions $g$ defined by

$$
\begin{aligned}
f(12) & =f(1) f(2)[1+g(12)] \\
f(123) & =f(1) f(2) f(3)[1+g(12)+g(13)+g(23)+g(123)]
\end{aligned}
$$

By neglecting $g(12)$ in Eq. (10a), we find that Eq. (8a) reduces to the ordinary nonlinear Boltzmann equation. Here, however, we retain $g(12)$ in the first equation, but we truncate the hierarchy equations at a later stage by neglecting $g(123)$ in (10b).

Our intention is to take into account only the contributions of uncorrelated binary collision events and of ring collision events. The contributions from the uncorrelated binary collision events are contained in the nonlinear Boltzmann equation, which provides a foundation for the theory of transport processes in a dilute gas. The ring events are responsible for the leading divergencies that appear in the expansion of the corrections to the Boltzmann equation in powers of the density $n$. In order to obtain a meaningful kinetic theory for gases at moderate densities, where corrections to the Boltzmann equation need to be taken into account, one collects and sums together all contributions from ring events to obtain a new resummed collision operator. In the theory based on the BBGKY equations, this resummed operator can be easily obtained by neglecting $g(123)$ in Eq. (10b) and then solving Eq. (8b) 
for $g(12)$. In so doing, we ignore the contributions from other dynamical events, which in each order in the density are less divergent than the ring events. Thus, the approximation of neglecting $g(123)$, as well as the use of point $T$-operators, has as a consequence the fact that any corrections to the Boltzmann equation obtained here should be determined only to lowest order in the density. A similar situation is also encountered in the linear theory discussed by Ernst and Dorfman, which is generalized here straightforwardly to include nonlinear effects.

If we then neglect $g(123)$ in Eq. (10b), we obtain the following closed set of equations ${ }^{(20)}$ :

$$
\begin{aligned}
& \left(\partial_{t}+\mathbf{v}_{1} \cdot \frac{\partial}{\partial \mathbf{r}_{1}}\right) f(1)=\int d x_{2} T(12) f(1) f(2)[1+g(12)] \\
& {\left[\partial_{t}+\mathbf{v}_{1} \cdot \frac{\partial}{\partial \mathbf{r}_{1}}+\mathbf{v}_{2} \cdot \frac{\partial}{\partial \mathbf{r}_{2}}-T(12)\right.} \\
& \left.\quad-\left(1+P_{12}\right) \int d x_{3} T(13)\left(1+P_{13}\right) f(3)\right] f(1) f(2) g(12) \\
& \quad=T(12) f(1) f(2)
\end{aligned}
$$

We are interested in the behavior of the distribution functions $f$ and $g$ for small gradients in the local variables, such as density $n$ and temperature $T=\left(k_{\mathrm{B}} \beta\right)^{-1}$, where $k_{\mathrm{B}}$ is Boltzmann's constant. In this paper we want to restrict ourselves to one type of gradient only, namely off-diagonal elements, $X=\nabla_{x} u_{y}$, of the rate of strain tensor, and we will evaluate the elements of the pressure tensor $P_{\alpha \beta}(\alpha, \beta=x, y, z)$. All remaining gradients vanish, so that $n$ and $T$ are uniform, and $\nabla \cdot \mathbf{u}=0$. Hence, we are dealing with uniform shear flow. In this approximation all local variables are stationary, as follows from Euler's equations, and $\partial_{t} f=\partial_{t} g=0$. By imposing this steady state we are ignoring the viscous heating of the fluid, i.e., $\partial_{t} T \propto \eta X^{2}$. In the discussion we will briefly return to this point.

In lowest approximation the distribution functions are denoted by $f_{0}(1)$ and $g_{0}(12)$, and have the local equilibrium form

$$
f_{0}(1)=n(\beta m / 2 \pi)^{d / 2} \exp \left\{-\frac{1}{2} \beta m\left[\mathbf{v}_{1}-\mathbf{u}\left(\mathbf{r}_{1}\right)\right]^{2}\right\}, \quad g_{0}(12)=0
$$

One might expect that the local equilibrium pair correlation function $g_{0}(12)$ would be nonvanishing (and equal to -1 ) inside a sphere $r_{12}<\sigma$. Consistency with the use of point $T$-operators requires that this sphere shrinks to zero.

We now consider the next dominant terms in $f(1) \equiv f_{0}(1)[1+h(1)]$ and $g(12)$ for small $X$. For that purpose we separate the unknown functions $h$ and $g$ into regular parts $h_{R}$ and $g_{R}$ (i.e., expandable in powers of $X$ around $X=0$ ), 
which start off proportional to $X$, and singular parts $\delta h$ and $\delta g$, which have no power series expansion in $X$ around $X=0$, i.e.,

$$
\begin{aligned}
f(1) & =f_{0}(1)\left[1+X h_{R}(1)+\delta h(1)\right] \\
g(12) & =X g_{R}(12)+\delta g(12)
\end{aligned}
$$

For the following arguments it is immaterial whether the singular terms are more (as for $d=2$ ) or less (as for $d=3$ ) dominant than $O(X)$ ). The ordering procedure for solving Eqs. (11a) and (11b) is as follows. Since we are interested in the leading singularities in $\delta h$ and $\delta g$, we keep in (11a) and (11b) only the most dominant regular [of $O\left(X \delta^{0}\right)$ ] and singular terms [of $O\left(X^{0} \delta\right)$ ]. However, since the "coefficient" of $\delta g(12)$ in Eq. (11b) can be vanishingly small to $O\left(X^{0}\right)$, (as will be shown explicitly later), we will also keep the $O(X)$ terms in this coefficient. This vanishing coefficient for small $X$ is precisely the source of the singular $X$ dependence of $\delta g$ and $\delta h$. The above ordering procedure ensures that our equations describe the leading singularities in $\delta h$ and $\delta g$ correctly.

Consider now Eq. (11a). Due to the presence of $\delta\left(\mathbf{r}_{12}\right)$ in $T(12)$, all local variables (i.e., here local velocities only) refer to the spatial position $\mathbf{r}_{1}$, and we will write henceforward $\mathbf{u}\left(\mathbf{r}_{1}\right)=\mathbf{u}$. Carrying out the above program yields

$$
\begin{aligned}
\beta J_{x y}\left(\mathbf{V}_{1}\right) X= & \Lambda\left(\mathbf{V}_{1}\right)\left[h_{R}\left(\mathbf{V}_{1}\right) X+\delta h\left(\mathbf{V}_{1}\right)\right] \\
& +n \int d \mathbf{V}_{2} \phi\left(V_{2}\right) T_{0}(12)\left[g_{R}\left(\mathbf{V}_{1} \mathbf{V}_{2} \mathbf{r}_{12}\right) X+\delta g\left(\mathbf{V}_{1} \mathbf{V}_{2} \mathbf{r}_{12}\right)\right]
\end{aligned}
$$

The functions $g_{R}$ and $\delta g$ depend also on the absolute position in space. However, it will be seen that this dependence is only parametric. We further introduced $\mathbf{V}_{i}=\mathbf{v}_{i}-\mathbf{u}$ [recall that $\mathbf{u}\left(\mathbf{r}_{1}\right)=\mathbf{u}$ ], and

$$
\begin{aligned}
\phi(V) & =(\beta m / 2 \pi)^{d / 2} \exp \left(-\frac{1}{2} \beta m V^{2}\right) \\
J_{\alpha \beta}(\mathbf{V}) & =m\left[V_{\alpha} V_{\beta}-(1 / d) \delta_{\alpha \beta} V^{2}\right] \\
\Lambda\left(\mathbf{V}_{1}\right) & =n \int d \mathbf{V}_{2} \phi\left(V_{2}\right) T_{0}(12)\left(1+P_{12}\right)
\end{aligned}
$$

where $\Lambda(v)$ is the linearized Boltzmann collision operator. We have treated $h$ and $g$ as functions that depend on the local velocity only in the combination $\mathbf{V}_{i}=\mathbf{v}_{i}-\mathbf{u}$. This is a consequence of the fact that the terms present in Eq. (14), and also in the equation for $g(12)$ to be derived below, depend only on this combination. Therefore, $h$ and $g$ depend on the absolute position in space only through $\mathbf{u}$, which enters only in the velocity variables $\mathbf{V}_{i}$.

The regular and singular parts satisfy Eq. (14) separately. The regular 
part is equal to the sum of two terms $h_{\mathrm{B}}+h_{B^{\prime}}$, where $h_{\mathrm{B}}(\mathrm{V})$ is the ChapmanEnskog solution of the Boltzmann equation, i.e.,

$$
\Lambda(\mathbf{v}) h_{\mathrm{B}}(\mathbf{v})=\beta J_{x y}(\mathbf{v})
$$

and $\Lambda(\mathbf{v}) h_{R^{\prime}}(\mathbf{v})$ equals an integral involving $g_{R}$. The functions $h_{R^{\prime}}$ and $g_{R}$ represent higher density corrections, which from now on will be neglected in Eq. (14) with respect to $h_{\mathrm{B}}(\mathbf{v})$ for consistency; that is, we replace $h_{R}$ by $h_{\mathrm{B}}$ everywhere.

The equation for the singular part becomes, after introducing a Fourier representation for the variable $\mathbf{r}_{12}$,

$$
\Lambda\left(\mathbf{v}_{1}\right) \delta h\left(\mathbf{v}_{1}\right)=-n \int \frac{d \mathbf{k}}{(2 \pi)^{d}} \int d \mathbf{v}_{2} \phi\left(v_{2}\right) T_{0}(12) \delta G\left(\mathbf{v}_{1} \mathbf{v}_{2} \mathbf{k}\right)
$$

where

$$
\delta G\left(\mathbf{v}_{1} \mathbf{v}_{2} \mathbf{k}\right)=\int d \mathbf{r}_{12}\left[\exp \left(-i \mathbf{k} \cdot \mathbf{r}_{12}\right)\right] \delta g\left(\mathbf{v}_{1} \mathbf{v}_{2} \mathbf{r}_{12}\right)
$$

An equation for $\delta G$ can be derived from (11b) in essentially the same manner. Since the calculations are somewhat more involved, they are carried out in Appendix A. The result is

$$
\begin{aligned}
& {\left[i \mathbf{k} \cdot \mathbf{v}_{12}-\Lambda\left(\mathbf{v}_{1}\right)-\Lambda\left(\mathbf{v}_{2}\right)+X R\left(\mathbf{v}_{1} \mathbf{v}_{2} \mathbf{k}\right)\right] \delta G\left(\mathbf{v}_{1} \mathbf{v}_{2} \mathbf{k}\right)} \\
& \quad=X T_{0}(12)\left(1+P_{12}\right) h_{\mathrm{B}}\left(\mathbf{v}_{1}\right)
\end{aligned}
$$

The explicit form of the operator $R\left(\mathbf{v}_{1} \mathbf{v}_{2} \mathbf{k}\right)$ can be found in Eq. (A14) of Appendix A. It is even in the variables $\mathbf{v}_{1}, \mathbf{v}_{2}$, and $\mathbf{k}$.

It is then implied by Eq. (19) that

$$
G\left(\mathbf{v}_{1}, \mathbf{v}_{2}, \mathbf{k}\right)=G\left(\mathbf{v}_{2}, \mathbf{v}_{1},-\mathbf{k}\right)=G\left(-\mathbf{v}_{2},-\mathbf{v}_{1}, \mathbf{k}\right)
$$

From the solutions of Eqs. (16)-(19) we can calculate the dissipative part $\boldsymbol{P}_{\alpha \beta}$ of the pressure tensor,

$$
P_{\alpha \beta}=n\left\langle J_{\alpha \beta}\left(h_{\mathrm{B}}+\delta h\right)\right\rangle
$$

The angular brackets denote an average over one velocity variable, defined as $\langle f(\mathbf{v})\rangle=\int d \mathbf{v} \phi(v) f(\mathbf{v})$. The singular part of the pressure tensor $\delta P_{\alpha \beta}$ is found from Eqs. (17) and (21)

$$
\delta P_{\alpha \beta}=n\left\langle J_{\alpha \beta} \delta h\right\rangle=-n^{2} \int \frac{d \mathbf{k}}{(2 \pi)^{d}}\left\langle\left\langle J_{\alpha \beta}\left(\mathbf{v}_{1}\right) \Lambda^{-1}\left(\mathbf{v}_{1}\right) T_{0}(12) \delta G\left(\mathbf{v}_{1} \mathbf{v}_{2} \mathbf{k}\right)\right\rangle\right\rangle
$$

The double brackets denote an average over two velocity variables. Only in two-dimensional systems is the leading contribution to Eqs. (19) and (22) for small $X$ actually a singular term in the pressure tensor. In three-dimensional systems the leading contributions are proportional to $X$, and should be 
subtracted out before the singular term is obtained. We will return to this point in Section 4 and Appendix B.

\section{REDUCTION TO SETS OF LINEAR DIFFERENTIAL EQUATIONS}

Let us expand $\delta G\left(\mathbf{v}_{1} \mathbf{v}_{2} \mathbf{k}\right)$ in eigenfunctions ${ }^{6} \psi_{\mathbf{k}}{ }^{\lambda}(\mathbf{v})$ of the linear Boltzmann operator $\{i \mathbf{k} \cdot \mathbf{v}-\Lambda(\mathbf{v})\}$ with eigenvalue $-z_{k}{ }^{\lambda}$, satisfying $\left\langle\psi_{\mathbf{k}}{ }^{\lambda} \psi_{\mathbf{k}}{ }^{p}\right\rangle=\delta_{\lambda v}$. Then

$$
\delta G\left(\mathbf{v}_{1} \mathbf{v}_{2} \mathbf{k}\right)=-\frac{1}{n} X \sum_{\lambda \mu} \psi_{\mathbf{k}}^{\lambda}\left(\mathbf{v}_{1}\right) \psi_{-\mathbf{k}}^{\mu}\left(\mathbf{v}_{2}\right) B^{\lambda \mu}(\mathbf{k})
$$

The factor $-X / n$ is taken out for later convenience. The first terms in Eq. (19) are then

$$
\left[i \mathbf{k} \cdot \mathbf{v}_{12}-\Lambda\left(\mathbf{v}_{1}\right)-\Lambda\left(\mathbf{v}_{2}\right)\right] \psi_{\mathbf{k}}^{\lambda}\left(\mathbf{v}_{1}\right) \psi_{-\mathbf{k}}^{\mu}\left(\mathbf{v}_{2}\right)=-\left(z_{k}^{\lambda}+z_{k}^{\mu}\right) \psi_{\mathbf{k}}^{\lambda}\left(\mathbf{v}_{1}\right) \psi_{-\mathbf{k}}^{\mu}\left(\mathbf{v}_{2}\right)
$$

The sum of eigenvalues may be of $O\left(k^{2}\right)$ for small $k$ and for certain combinations of hydrodynamic modes. We choose a sufficiently small cutoff wave number $k_{0} \propto n \sigma^{d-1}$ (on the order of the inverse mean free path), such that the $\mathbf{k}$ expansion of eigenvalues and eigenfunctions holds for all $k<k_{0}$. An inspection of Eq. (19) then suggests that for small $k$ and $X$ the resulting expression $\delta G\left(\mathbf{v}_{1} \mathbf{v}_{2} \mathbf{k}\right)$ would contain terms of the form $\delta G \sim\left(k^{2}+X\right)^{-1}$, and $\delta P$ in Eq. (22) would then contain $k$ integrals of the form $\int_{0}^{k_{0}} d k k^{d-1}\left(k^{2}+X\right)^{-1}$. It is clear that this expression does not exist in two dimensions at $X=0$, and that its derivative at $X=0$ does not exist for three-dimensional systems. On the other hand, the presence of $O(X)$ terms may protect the dangerous denominators at the lower limit of the $k$ integration, so that $\delta P_{\alpha \beta} / X$ does exist, but is a singular function of $X$ at $X=0$.

The contributions from nonhydrodynamic modes, as well as from large $k$ values, and those from hydrodynamic modes with $z_{k}{ }^{\lambda}+z_{k}{ }^{\mu}=O(k)$ do exist in two and three dimensions at $X=0$, and their first derivatives exist also in three dimensions at $X=0$ (or are at least less singular). Such contributions should be lumped together in $G_{R}$, which should be neglected for consistency, since it is a higher density correction with respect to the right-hand side of (19).

After having explained the general procedure, we list the explicit expressions for the hydrodynamic modes, i.e., sound modes $(\sigma= \pm)$, heat mode

${ }^{6}$ The operator $i \mathbf{k} \cdot \mathbf{v}-\Lambda(\mathbf{v})$ is non-Hermitian (but it is symmetric). One should therefore consider biorthogonal sets of right eigenfunctions $\psi_{\mathbf{k}}{ }^{\mu}$ and left eigenfunctions $\tilde{\psi}_{\mathrm{k}}{ }^{\lambda}$. However, since $\tilde{\psi}_{\mathbf{k}}{ }^{\lambda}$ is the complex conjugate of $\psi_{\mathbf{k}}{ }^{\lambda}$, one can write inner products as $\left(\tilde{\psi}_{\mathbf{k}}{ }^{\lambda}, \psi_{\mathbf{k}}{ }^{\mu}\right)=\left\langle\tilde{\psi}_{\mathbf{k}}^{\lambda^{*}} \psi_{\mathbf{k}}{ }^{\mu}\right\rangle=\left\langle\psi_{\mathbf{k}}{ }^{\lambda} \psi_{\mathbf{k}}{ }^{\mu}\right\rangle$. As we will restrict our attention only to hydrodynamic modes, it will not be important if $i \mathrm{k} \cdot \mathrm{v}-\Lambda$ (v) does not have a complete (biorthogonal) set of eigenfunctions. 
$(T)$, and $(d-1)$ shear modes $\left(\eta_{i} ; i=1,2, \ldots, d-1\right)$, which are only needed to $O\left(k^{0}\right)$ :

$$
\begin{aligned}
& \psi_{\mathbf{k}}^{\sigma}(\mathbf{v})=\psi_{-\mathbf{k}}^{-\sigma}(\mathbf{v})=\frac{\beta m v^{2}}{[2 d(d+2)]^{1 / 2}}+\sigma\left(\frac{\beta m}{2}\right)^{1 / 2} \mathbf{v} \cdot \hat{\mathbf{k}} \\
& \psi_{\mathbf{k}}^{T}(\mathbf{v})=\psi_{-\mathbf{k}}^{T}(\mathbf{v})=\frac{1}{[2(d+2)]^{1 / 2}}\left(\beta m v^{2}-d-2\right) \\
& \psi_{\mathbf{k}}^{n_{i}}(\mathbf{v})=\operatorname{sg}(i) \psi_{-\mathbf{k}}^{n_{n}}(\mathbf{v})=(\beta m)^{1 / 2} \hat{\mathbf{k}}_{\perp}^{(i)} \cdot \mathbf{v}
\end{aligned}
$$

We choose $\hat{\mathbf{k}}_{\perp}^{(i)}$ in such a way that $(-\hat{\mathbf{k}})_{\perp}^{(i)}=\operatorname{sg}(i) \hat{\mathbf{k}}_{\perp}^{(i)}$, where $\operatorname{sg}(i)$ is +1 or -1 , and will be chosen later on. The hydrodynamic frequencies are

$$
z_{k}{ }^{\sigma}=-i \sigma c_{0} k-D_{\mathrm{s}} k^{2} ; \quad z_{k}{ }^{T}=-D_{T} k^{2} ; \quad z_{k}^{\eta_{i}}=-D_{\eta} k^{2}
$$

Here, $\left(\hat{\mathbf{k}}, \hat{\mathbf{k}}_{\perp}^{(1)}, \hat{\mathbf{k}}_{\perp}^{(2)}, \ldots\right)$ is a set of mutually orthogonal unit vectors; $c_{0}=[(d+2) / d \beta m]^{1 / 2}$ is the adiabatic sound velocity; $D_{s}=(1 / d) D_{T}+$ $[(d-1) / d] D_{\eta}$ is the sound damping constant, where the thermal diffusivity $D_{T}=\lambda / n C_{p}$ contains the heat conductivity $\lambda$ and the specific heat per particle at constant pressure $C_{p}=\frac{1}{2}(d+2) k_{\mathrm{B}}$; and $D_{\eta}=\eta / n m$ is the kinematic viscosity. The pairs of dangerous hydrodynamic modes are indicated by $\langle\lambda \mu\rangle=(\sigma,-\sigma),(T T),\left(\eta_{i} T\right),\left(T \eta_{i}\right),\left(\eta_{i} \eta_{j}\right)$. We now insert Eq. (23) into Eq. (19) and calculate the projection $\left\langle\left\langle\psi_{\mathbf{k}}{ }^{\lambda}\left(\mathbf{v}_{1}\right) \psi_{-\mathbf{k}}^{\mu}\left(\mathbf{v}_{2}\right)\right.\right.$ [Eq. (19)] $\left.\rangle\right\rangle$, with the result

$$
\sum_{\langle v \rho\rangle}\left[\delta_{\lambda v} \delta_{\mu \rho}\left(D_{\lambda}+D_{\mu}\right) k^{2}+X R_{v \rho}^{\lambda \mu}(\mathbf{k})\right] B^{v \rho}(\mathbf{k})=\beta A_{x y}^{\lambda \mu}(\mathbf{k})
$$

The right-hand side of Eq. (19) has been calculated to $O\left(k^{0}\right)$, using the relation

$$
\left\langle\left\langle\psi_{\mathbf{k}}{ }^{\lambda}\left(\mathbf{v}_{1}\right) \psi_{-\mathbf{k}}^{\mu}\left(\mathbf{v}_{2}\right) T_{0}(12)\left(1+P_{12}\right) h_{\mathrm{B}}\left(\mathbf{v}_{1}\right)\right\rangle\right\rangle=-(1 / n)\left\langle\psi_{\mathbf{k}}{ }^{\lambda} \psi_{-\mathbf{k}}^{\mu} \Lambda h_{\mathrm{B}}\right\rangle
$$

together with Eq. (16) and the definition

$$
A_{\alpha \beta}^{\lambda \mu}(\mathbf{k})=\left\langle\psi_{\mathbf{k}}{ }^{\lambda} \psi_{-\mathbf{k}}^{\mu} J_{\alpha \beta}\right\rangle
$$

We further introduced the matrix elements of $R\left(\mathbf{v}_{1} \mathbf{v}_{2} \mathbf{k}\right)$, defined in (A14),

$$
R_{v \rho}^{\lambda \mu}(\mathbf{k})=\left\langle\left\langle\psi_{\mathbf{k}}^{\lambda}\left(\mathbf{v}_{1}\right) \psi_{-\mathbf{k}}^{\mu}\left(\mathbf{v}_{2}\right) R\left(\mathbf{v}_{1} \mathbf{v}_{2} \mathbf{k}\right) \psi_{k}{ }^{v}\left(\mathbf{v}_{1}\right) \psi_{-\mathbf{k}}^{o}\left(\mathbf{v}_{2}\right)\right\rangle\right\rangle
$$

One should notice that $R_{v \rho}^{\lambda \mu}(\mathbf{k})$ is a differential operator with $d / d k_{x}$ acting on all k-dependent functions to its right. In this manner we have reduced the determination of $\delta G$ to the solution of a coupled set of linear first-order differential equations.

The singular part of the pressure tensor (22) can now be expressed as

$$
\delta P_{\alpha \beta}=-\frac{1}{2} X \int^{\prime} \frac{d \mathbf{k}}{(2 \pi)^{d}} \sum_{\langle\lambda \mu\rangle} A_{\alpha \beta}^{\lambda \mu}(\mathbf{k}) B^{\lambda \mu}(\mathbf{k})
$$

where we have used Eqs. (28)-(29) and the symmetry relations, implied by Eq. (20),

$$
B^{\lambda \mu}(\mathbf{k})=B^{\mu \lambda}(-\mathbf{k})
$$


and for future reference we also list the symmetry properties, implied by Eqs. (25), (27), and (20), i.e.,

$$
\begin{gathered}
B^{+-}(\mathbf{k})=B^{-+}(\mathbf{k}), \quad B^{\eta_{i} T}(\mathbf{k})=-\operatorname{sg}(i) B^{T \eta_{i}}(\mathbf{k}) \\
B^{\eta_{i} \eta_{j}}(\mathbf{k})=\operatorname{sg}(i) \operatorname{sg}(j) B^{\eta_{j} \eta_{i}}(\mathbf{k})
\end{gathered}
$$

Before solving Eq. (27) we have to calculate the amplitudes $A_{\alpha \beta}^{\lambda \mu}(\mathbf{k})$ and the matrix elements $R_{v \rho}^{\lambda \mu}(\mathbf{k})$. They are only needed to $O\left(k^{0}\right)$, since $O\left(X k^{0}\right)$ terms provide the main protection mechanism against the divergences caused by the $O\left(k^{2} X^{0}\right)$ terms. For the amplitudes we find from Eqs. (25) and (29)

$$
\begin{gathered}
\beta A_{\alpha \beta}^{\sigma-\sigma}=\hat{k}_{\alpha} \hat{k}_{\beta}-(1 / d) \delta_{\alpha \beta}, \quad A_{\alpha \beta}^{T T}=A_{\alpha \beta}^{\eta_{1} T}=A_{\alpha \beta}^{T \eta_{i}}=0 \\
\beta A_{\alpha \beta}^{\eta_{i} \eta_{j}}=\operatorname{sg}(j)\left(\hat{k}_{\perp \alpha}^{(i)} \hat{k}_{\perp \beta}^{(j)}+\hat{k}_{\perp \beta}^{(i)} \hat{k}_{\perp \alpha}^{(j)}-(2 / d) \delta_{\alpha \beta} \delta_{i j}\right)
\end{gathered}
$$

The matrix elements of (30) to $O\left(k^{0}\right)$ are given in Eqs. (A14) of Appendix A and read

$$
\begin{aligned}
R_{v \rho}^{\lambda \mu}(\mathbf{k})= & \left\langle\left\langle\psi _ { \mathbf { k } } ^ { \lambda } ( \mathbf { v } _ { 1 } ) \psi _ { - \mathbf { k } } ^ { u } ( \mathbf { v } _ { 2 } ) \left(-\frac{d}{d k_{x}} k_{y}-v_{1 x} \frac{d}{d v_{1 y}}-v_{2 x} \frac{d}{d v_{2 y}}\right.\right.\right. \\
& \left.\left.\left.+\beta m v_{1 x} v_{1 y}+\beta m v_{2 x} v_{2 y}\right) \psi_{\mathbf{k}}{ }^{\nu}\left(\mathbf{v}_{1}\right) \psi_{-\mathbf{k}}^{\rho}\left(\mathbf{v}_{2}\right)\right\rangle\right\rangle
\end{aligned}
$$

Lengthy but straightforward calculations yield explicit expressions for the matrix elements in $d$-dimensional systems to zeroth order in $k$,

$$
\begin{aligned}
& R_{\sigma^{\prime}-\sigma^{\prime}}^{\sigma-\sigma}(\mathbf{k})=\delta_{\sigma \sigma^{\prime}}\left[-\left(d / d k_{x}\right) k_{y}+\hat{k}_{x} \hat{k}_{y}\right] \\
& R_{\eta_{j} T}^{n_{1} T}(\mathbf{k})=\operatorname{sg}(i) \operatorname{sg}(j) R_{T \eta_{j}}^{T \eta_{i}}(\mathbf{k}) \\
& =\hat{k}_{\perp y}^{(i)} \hat{k}_{\perp x}^{(j)}-\hat{k}_{\perp \alpha}^{(i)}\left(d / d k_{x}\right) k_{y} \hat{k}_{\perp \alpha}^{(j)} \\
& R_{n_{j} \eta_{j^{\prime}}}^{n_{n_{i} i^{\prime}}}(\mathbf{k})=\operatorname{sg}\left(i^{\prime}\right) \operatorname{sg}\left(j^{\prime}\right)\left[\delta_{i^{\prime} j^{\prime}} \hat{k}_{\perp y}^{(i)} \hat{k}_{\perp x}^{(j)}+\delta_{i j} \hat{k}_{\perp y}^{\left(i^{\prime}\right)} \hat{k}_{\perp x}^{\left(j^{\prime}\right)}\right. \\
& \left.-\hat{k}_{\perp \alpha}^{(i)} \hat{k}_{\perp \beta}^{\left(i^{\prime}\right)}\left(d / d k_{x}\right) k_{y} \hat{k}_{\perp \alpha}^{(j)} \hat{k}_{\perp \beta}^{\left(j^{\prime}\right)}\right] \\
& R_{T T}^{T T}(\mathbf{k})=-\left(d / d k_{x}\right) k_{y} \\
& R_{v \rho}^{\lambda \mu}=0 \quad \text { (in all other cases of interest) }
\end{aligned}
$$

Summation convention is used for repeated indices.

It is obvious from Eqs. (36) and (26) that the differential equation for $B^{+-}(\mathbf{k})$ in $d$ dimensions does not couple to any other $B^{\lambda \mu}(\mathbf{k})$, and we have for the sound mode contributions

$$
\left[2 D_{s} k^{2}-X k_{y}\left(d / d k_{x}\right)+X \hat{k}_{x} \hat{k}_{y}\right] B^{+-}(\mathbf{k})=\hat{k}_{x} \hat{k}_{y}
$$

Equations (34) and (31) require further that $B^{n_{i} n_{j}}(\mathbf{k})$ be determined. In two dimensions, where $\hat{\mathbf{k}}=\left(\hat{k}_{x}, \hat{k}_{y}\right)$ and $\hat{\mathbf{k}}_{\perp}=\left(-\hat{k}_{y}, \hat{k}_{x}\right)$ [hence $\operatorname{sg}(1)=-1$ in two dimensions], the equation for the shear mode contributions becomes

$$
\left[2 D_{n} k^{2}-X k_{y}\left(d / d k_{x}\right)-2 X \hat{k}_{x} \hat{k}_{y}\right] B^{n_{1} n_{1}}(\mathbf{k})=2 \hat{k}_{x} \hat{k}_{y}
$$


upon using Eqs. (27), (34), (36), and (31) and the relation $\hat{k}_{\perp \alpha}\left(d / d k_{x}\right) \hat{k}_{\perp \alpha}=0$. In three dimensions the equation for the shear mode contributions can be simplified enormously by choosing a special coordinate system ${ }^{(21)}$ such that $d \hat{k}_{\perp \alpha}^{(2)} / d k_{x}=0$, which implies

$$
\hat{k}_{\perp \alpha}^{(1)}\left(d / d k_{x}\right) \hat{k}_{\perp \alpha}^{(2)}=-\hat{k}_{\perp \alpha}^{(2)}\left(d / d k_{x}\right) \hat{k}_{\perp \alpha}^{(1)}=0
$$

This leads to the following set of unit vectors:

$$
\begin{aligned}
\hat{\mathbf{k}} & =\left(\hat{k}_{x}, \hat{k}_{y}, \hat{k}_{z}\right) \\
\hat{\mathbf{k}}_{\perp}^{(1)} & =\left(-\hat{k}_{y}{ }^{2}-\hat{k}_{z}{ }^{2}, \hat{k}_{x} \hat{k}_{y}, \hat{k}_{x} \hat{k}_{z}\right) /\left(\hat{k}_{y}{ }^{2}+\hat{k}_{z}{ }^{2}\right)^{1 / 2} \\
\hat{\mathbf{k}}_{\perp}^{(2)} & =\left(0,-\hat{k}_{z}, \hat{k}_{y}\right) /\left(\hat{k}_{y}{ }^{2}+\hat{k}_{z}{ }^{2}\right)^{1 / 2}
\end{aligned}
$$

with $\operatorname{sg}(1)=1$ and $\operatorname{sg}(2)=-1$.

The choice (40) enables us to write down expressions for $R_{n_{j} \eta_{j^{\prime}}}^{n_{1} \eta_{n^{\prime}}}$ in terms of $\hat{k}_{x}, \hat{k}_{y}$, and $\hat{k}_{z}$, and we find the following set of differential equations for the shear mode contributions:

$$
\begin{aligned}
& {\left[2 D_{n} k^{2}-X k_{y}\left(d / d k_{x}\right)-2 X \hat{k}_{x} \hat{k}_{y}\right] B^{n_{1} n_{1}}(\mathbf{k})=-2 \hat{k}_{x} \hat{k}_{y}} \\
& {\left[2 D_{\eta} k^{2}-X k_{y}\left(d / d k_{x}\right)-X \hat{k}_{x} \hat{k}_{y}\right] B^{n_{2} n_{1}}(\mathbf{k})=\hat{k}_{z}\left\{1-X B^{n_{1} n_{1}}(\mathbf{k})\right\}} \\
& \left(2 D_{\eta} k^{2}-X k_{y}\left(d / d k_{x}\right)\right) B^{n_{z} \eta_{2}}(\mathbf{k})=2 X \hat{k}_{z} B^{\eta_{2} \eta_{1}(\mathbf{k})}
\end{aligned}
$$

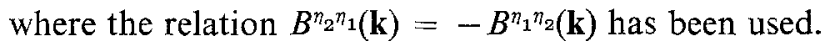

In order to construct a unique solution of Eqs. (37), (38), or (41) we must impose a boundary condition on $B^{\lambda \mu}(\mathbf{k}, X)$. We note that it is possible to obtain a solution to each of these equations as an expansion in powers of $X$, by iterating about the $X=0$ solution. For example, Eq. (37) has the iterated solution

$$
B^{+-}(\mathbf{k})=B_{0}(\mathbf{k})+X B_{1}(\mathbf{k})+X^{2} B_{2}(\mathbf{k})+\cdots
$$

with

$$
B_{0}(\mathbf{k})=\left(2 D_{s} k^{2}\right)^{-1} \hat{k}_{x} \hat{k}_{y}
$$

and $B_{i}(\mathbf{k})$ determined by the relation

$$
B_{i}(\mathbf{k})=\left(2 D_{s} k^{2}\right)^{-1}\left[k_{y}\left(d / d k_{x}\right)-\hat{k}_{x} \hat{k}_{y}\right] B_{i-1}(\mathbf{k})
$$

The expansion of $B^{+-}(\mathbf{k})$ in powers of $X$ leads to an expansion of the pressure tensors in powers of $X$, and we see by considering Eqs. (31) and (42) that the coefficient of each power of $X$ in this expression of $\delta P_{\alpha \beta}$ is divergent, with the degree of divergence increasing with each power of $X$. It is this divergent expansion that we want to resum. To carry out the resummation we must resum Eq. (42a) for $B^{+-}(\mathbf{k})$ and the corresponding equation for $B^{n_{i} n_{j}}(\mathbf{k})$ before carrying out the final $k$ integration. The differential equations (37), (38), and (41) provide a means by which this summation may be readily 
carried out. We merely need to construct solutions to these differential equations that, upon expansion in powers of $X$, agree with the expansion (42a) or the corresponding ones for $B^{\eta_{i} \eta_{j}}(\mathbf{k})$. We also note that, since we are interested here only in the most singular part of $\delta P_{\alpha \beta}(X)$ for small $X$, we can add to the singular part of $B^{\lambda \mu}(\mathbf{k})$ any terms that are well behaved for small $k$ and $X$ without disturbing the singular part of $\delta P_{\alpha \beta}(X)$. Since the $X$-dependent terms are only important to modify the behavior of $B^{\lambda \mu}(\mathbf{k})$ at small $k$, we can impose the boundary condition that $B(\mathbf{k}, X)$ coincides with $B(\mathbf{k}, X=0)$ at some point on the outer boundary of the $\mathbf{k}$ integration. Combining these observations, we now choose the boundary conditions for $B^{\lambda \mu}(\mathbf{k})$ such that

$$
B^{\lambda \mu}\left(\mathbf{k}^{0}, X\right)=B^{\lambda \mu}\left(\mathbf{k}^{0}, X=0\right)=A_{x y}^{\lambda \mu}\left(\mathbf{k}^{0}\right)\left[\left(D_{\lambda}+D_{\mu}\right)\left(k^{0}\right)^{2}\right]^{-1}
$$

where the point $\mathbf{k}^{0}$ is chosen so that the singular parts of the $X$-expanded solutions of the differential equations agree with the iterated solutions. These requirements are met at the boundary point

$$
\mathbf{k}^{0}=\left(\theta k_{0}, k_{y}, k_{z}\right)
$$

where $\theta=\operatorname{sg}\left(X k_{y}\right)$ is the sign of $X k_{y}$.

\section{SINGULAR PARTS OF THE PRESSURE TENSOR}

In this section we will solve the differential equations (37), (38), and (41), and then complete the calculation of the singular part of the pressure tensor given in (31). We consider the two-dimensional case first. Inspection of Eqs. (37) and (38) shows that for two dimensions both relevant differential equations can be put in the general form

$$
\left(\frac{d}{d k_{x}}+n \frac{k_{x}}{k^{2}}-\theta \frac{g^{(n)} k^{2}}{\left|k_{y}\right|}\right) B^{(n)}(\mathbf{k})=\frac{1}{X} I^{(n)}(\mathbf{k})
$$

with $\theta=\operatorname{sg}\left(X k_{y}\right)$. By setting

$$
n=-1 ; \quad g^{(-1)}=2 D_{s} /|X| ; \quad I^{(-1)}=-k_{x} \mid k^{2} ; \quad B^{(-1)}=B^{+-}
$$

we recover Eq. (37), and by setting

$$
n=2 ; \quad g^{(2)}=2 D_{n} /|X| ; \quad I^{(2)}=-2 k_{x} / k^{2} ; \quad B^{(2)}=B^{\eta \eta}
$$

we recover Eq. (38). The corresponding boundary conditions, as discussed in Eqs. (43a) and (43b), are

$$
\begin{aligned}
& B^{(-1)}\left(\mathbf{k}^{0}, X\right)=B^{(-1)}\left(\mathbf{k}^{0}, 0\right)=\theta k_{0} k_{y} / 2 D_{s}\left(k^{0}\right)^{4} \\
& B^{(2)}\left(\mathbf{k}^{0}, X\right)=B^{(2)}\left(\mathbf{k}^{0}, 0\right)=2 \theta k_{0} k_{y} / 2 D_{\eta}\left(k^{0}\right)^{4}
\end{aligned}
$$


Equation (44) can be easily solved using an integrating factor, and the general solution is

$$
\begin{aligned}
B^{(n)}(\mathbf{k})= & \left(k^{0} / k\right)^{n} K^{(n)}\left(\theta \mathbf{k}, \theta \mathbf{k}^{0}\right) B^{(n)}\left(\mathbf{k}^{0}, 0\right) \\
& -(1 / X) \int_{k_{x}}^{\theta k_{0}} d k_{x}{ }^{\prime}\left(k^{\prime} / k\right)^{n} K^{(n)}\left(\theta \mathbf{k}, \theta \mathbf{k}^{\prime}\right) I^{(n)}\left(\mathbf{k}^{\prime}\right)
\end{aligned}
$$

where $\mathbf{k}^{\prime}=\left(k_{x}{ }^{\prime}, k_{y}\right)$ and

$$
K^{(n)}\left(\mathbf{k}, \mathbf{k}^{\prime}\right)=\exp \left\{g^{(n)} /\left|k_{y}\right|\left[k_{x}\left(k^{2}-\frac{2}{3} k_{x}{ }^{2}\right)-k_{x}{ }^{\prime}\left(k^{\prime 2}-\frac{2}{3} k_{x}{ }^{2}\right)\right]\right\}
$$

The singular part, Eq. (31), of the pressure tensor is now given by

$$
\delta P_{\alpha \beta}=-X \sum_{n=-1,2} \int^{\prime} \frac{d \mathbf{k}}{(2 \pi)^{2}} A_{\alpha \beta}^{(n)}(\mathbf{k}) B^{(n)}(\mathbf{k})
$$

with

$$
\begin{aligned}
A_{x y}^{(-1)} & =A_{x y}^{+-}=\beta^{-1} \hat{k}_{x} \hat{k}_{y}, \quad A_{x y}^{(2)}=\frac{1}{2} A_{x y}^{\eta \eta}=\beta^{-1} \hat{k}_{x} \hat{k}_{y} \\
A_{x x}^{(-1)} & =A_{x x}^{+-}=\beta^{-1}\left(\hat{k}_{x}^{2}-\frac{1}{2}\right)=-A_{y y}^{(-1)} \\
A_{x x}^{(2)} & =\frac{1}{2} A_{x x}^{\eta n}=\beta^{-1}\left(\hat{k}_{x}^{2}-\frac{1}{2}\right)=-A_{y y}^{(2)}
\end{aligned}
$$

We have also used the symmetry relation $B^{+-}(\mathbf{k})=B^{-+}(\mathbf{k})$ from Eqs. (32)-(33). The first term on the right-hand side of Eq. (48) gives a contribution $\delta P_{\alpha \beta}$ which remains finite as $|X| \rightarrow 0$, or equivalently, as $g^{(n)} \rightarrow \infty$. This contribution is a higher order density correction to the regular part of the viscous stresses, and is therefore neglected here.

The singular contribution to $\delta P_{\alpha \beta}$ comes from the second term on the right-hand side of Eq. (48), and insertion in Eq. (50) yields

$$
\begin{aligned}
\delta P_{\alpha \beta} & \equiv \sum_{n=-1,2} \delta P_{\alpha \beta}^{(n)} \\
& =\sum_{n=-1,2} 2 \int^{\prime \prime} \frac{d \mathbf{k}}{(2 \pi)^{2}} \int_{k_{x}}^{k_{0}} d k_{x}^{\prime}\left(\frac{k^{\prime}}{k}\right)^{n} K^{(n)}\left(\mathbf{k}, \mathbf{k}^{\prime}\right) I^{(n)}\left(\mathbf{k}^{\prime}\right) A_{\alpha \beta}^{(n)}(\mathbf{k})
\end{aligned}
$$

where the double prime on the $\mathbf{k}$ integration means that $|\mathbf{k}|<k_{0}$ and $X k_{y}>0$. We have further used the property

$$
(-) A_{\alpha \beta}^{(n)}(-\mathbf{k}) I^{(n)}\left(-\mathbf{k}^{\prime}\right)=A_{\alpha \beta}^{(n)}(\mathbf{k}) I^{(n)}\left(\mathbf{k}^{\prime}\right)
$$

Consider first the off-diagonal element. From Eqs. (51), (49), and (45) we obtain the sound mode contribution $(n=-1)$

$$
\begin{aligned}
\delta P_{x y}^{(-1)}= & -\frac{2}{\beta} \frac{\operatorname{sg}(X)}{(2 \pi)^{2}} \int_{0}^{k_{0}} d k k \int_{0}^{\pi} d \phi \int_{0}^{k-1 k_{0}-\hat{k}_{x}} d s \\
& \times \frac{\left(\hat{k}_{x}+s\right) \hat{k}_{x} \hat{k}_{y}}{\left(1+2 \hat{k}_{x} s+s^{2}\right)^{1 / 2}} \exp \left[-g^{(-1)} \hat{k}_{y}^{-1} s k^{2} E\left(\hat{k}_{x}, s\right)\right]
\end{aligned}
$$


To obtain the form given in Eq. (53) we have relabeled the variables $k_{x} \rightleftarrows-k_{x}{ }^{\prime}$ and introduced a new variable $s$ by $k_{x}{ }^{\prime}=k_{x}+s k$. In addition, we set $\hat{k}_{x}=\cos \phi$ and $\hat{k}_{y}=\sin \phi$, and $E\left(\hat{k}_{x} s\right)$ is given by

$$
E\left(\hat{k}_{x}, s\right)=1+\hat{k}_{x} s+\frac{1}{3} s^{2}
$$

The divergent integral, which occurs in the linear kinetic theory for $\delta \eta=-\delta P_{x y} / X$, can be obtained from Eq. (54) by making the substitution $s=|X| y$ and taking the limit $|X| \rightarrow 0$ inside the integrals. To evaluate the integral for $X \neq 0$, we interchange the $k$ and $s$ integrations and note that, since $k^{-1} k_{0}-\hat{k}_{x} \geqslant 1-\hat{k}_{x}$, we can write

$$
\int_{0}^{k_{0}} d k \int_{0}^{k^{-1} k_{0}-\hat{k}_{x}} d s \cdots=\int_{0}^{1-\hat{k}_{x}} d s \int_{0}^{k_{0}} d k \cdots+\int_{1-\hat{k}_{x}}^{\infty} d s \int_{0}^{k_{0}\left(s+k_{x}\right)-1} d k \cdots
$$

It can be easily seen that the region of integration $\left\{1-\hat{k}_{x} \leqslant s \leqslant \infty\right.$; $\left.0<k<k_{0}\left(s+\hat{k}_{x}\right)^{-1}\right\}$ leads to a contribution to $\delta P_{x y}^{(-1)}$ that is of order $X$ as $X \rightarrow 0$, and can be ignored here.

In the remaining region of integration $\left\{0 \leqslant s \leqslant 1-\hat{k}_{x} ; 0 \leqslant k \leqslant k_{0}\right\}$ we split up the $s$ integration into region $A=\left\{0 \leqslant s \leqslant s_{0}\right\}$ and region $B=$ $\left\{s_{0} \leqslant s \leqslant 1-\hat{k}_{x}\right\}$, where $s_{0}$ is chosen such that $g^{(-1)} s_{0} k_{0}{ }^{2}=n_{0} \ll 1$. It is easy to verify that the contribution to $\delta P_{x y}^{-(1)}$ from region $A$ is well behaved in $X$ for small $X$, and that the contribution from region $B$ is singular for small $X$, and is given by

$$
\begin{aligned}
\delta P_{x y}^{(-1)} & \simeq-\frac{X}{2 D_{s} \beta(2 \pi)^{2}} \int_{0}^{\pi} d \phi \int_{s_{0}}^{1-\hat{k}_{x}} d s \frac{\left(\hat{k}_{x}+s\right) \hat{k}_{x} \hat{k}_{y}{ }^{2}}{s E\left(\hat{k}_{x}, s\right)\left(1+2 \hat{k}_{x} s+s^{2}\right)^{1 / 2}} \\
& \simeq-\frac{1}{32 \pi \beta} \frac{1}{2 D_{\mathrm{s}}} X \log \frac{k_{0}{ }^{2} D_{s}}{|X|}
\end{aligned}
$$

where we have changed $s$ into $g^{(-1)} y$ and taken the dominant term as $X \rightarrow 0$ or $g^{(-1)} \rightarrow \infty$.

An almost identical calculation yields for the shear mode contribution

$$
\delta P_{x y}^{(2)} \simeq-\frac{1}{32 \pi \beta} \frac{1}{D_{\eta}} X \log \frac{D_{\eta} k_{0}^{2}}{|X|}
$$

Since the arguments of the logarithms in Eqs. (57) and (58) differ only a factor of $O(1)$, we may combine both terms into

$$
\delta P_{x y} \equiv-X \delta \eta(X) \simeq-\frac{1}{32 \pi \beta}\left(\frac{1}{D_{\eta}}+\frac{1}{2 D_{s}}\right) X \log \frac{D_{\eta} k_{0}{ }^{2}}{|X|}
$$

For two-dimensional systems at low density this is the leading singularity as $X \rightarrow 0$ of the nonlinear shear viscosity. 
Table I. Numerical Values of the Coefficient $M$ in Units $10^{-2}$

\begin{tabular}{llllll}
\hline$M_{\alpha \beta}$ & $M^{+-}$ & $M^{(2)}$ & $M^{(1)}$ & $M^{(0)}$ & $M^{\eta \eta}$ \\
\hline$x y$ & -0.406 & -0.766 & +0.507 & 0 & -0.259 \\
$x x$ & -0.329 & -1.79 & 0 & +9.38 & +7.59 \\
$y y$ & +0.287 & +0.759 & +0.693 & -14.73 & -13.28 \\
$z z$ & +0.042 & +1.03 & -0.693 & +5.36 & +5.70 \\
\hline
\end{tabular}

Next we consider the contributions $\delta P_{x x}^{(n)}$ and $\delta P_{y y}^{(n)}$ in Eq. (50), which are all vanishing, as direct computation shows, i.e.,

$$
\delta P_{x x}=\delta P_{y y}=0
$$

In three-dimensional systems we have obtained the following results for the singular parts of the pressure tensor in the case of uniform shear flow, i.e., $u_{\alpha}(\mathbf{r})=\delta_{\alpha y}\left(u_{0}+x X\right)$; for the shear stresses

$$
\begin{aligned}
& \delta P_{x y} \equiv-X \delta \eta(X)=-\frac{X|X|^{1 / 2}}{\beta}\left[\frac{M_{x y}^{\eta \eta}}{\left(2 D_{\eta}\right)^{3 / 2}}+\frac{M_{x y}^{+-}}{\left(2 D_{s}\right)^{3 / 2}}\right] \\
& \delta P_{x z}=\delta P_{y z}=0
\end{aligned}
$$

and for the normal stresses $(a=x, y, z)$

$$
\delta P_{a a}=-\frac{|X|^{3 / 2}}{\beta}\left[\frac{M_{a a}^{\eta n}}{\left(2 D_{\eta}\right)^{3 / 2}}+\frac{M_{a a}^{+-}}{\left(2 D_{s}\right)^{3 / 2}}\right]
$$

where $\sum_{a} \delta P_{a a}=0$. The coefficients

$$
M^{\eta \eta}=M^{(2)}+M^{(1)}+M^{(0)} ; \quad M^{+-}=M^{(-1)}
$$

are pure numbers, and represent respectively the contributions from the shear modes and opposite sound modes. The detailed calculations are given in Appendix B, and the numerical values of $M$ are given in Table I. In the next section these results will be discussed and compared with those of other investigators.

\section{CONCLUSION}

We conclude with a number of remarks:

1. In the case of a steady-state shear flow with a local velocity field $u_{\alpha}(\mathbf{r})=\delta_{\alpha y}\left(u_{0}+x X\right)$, one would traditionally expand the pressure tensor, e.g., its $x y$ element $P_{x y}$, in a power series in the velocity gradient $X$,

$$
P_{x y}=-\eta X-\eta^{\prime} X^{3}+\cdots
$$

As is well known, certain expansion coefficients do not seem to exist: In twodimensional systems the Navier-Stokes transport coefficient $\eta$ appears to be 
infinite; in three-dimensional systems the super-Burnett coefficient $\eta^{\prime}$ appears to be infinite. ${ }^{(8-12)}$ Here we presented a method that leads to a set of wellbehaved nonlinear equations for a simple steady-state shear flow with parallel plate geometry. Thus we have succeeded in renormalizing the divergence difficulties that occur in Eq. (64), and we have shown that when nonlinear effects of the velocity field are taken into account in computing the contribution of the ring events to the $x y$ element of the pressure tensor, one obtains in two-dimensional systems an expansion of $P_{x y}(X)$ of the form

$$
P_{x y}(X)=-X \eta(X)
$$

with

$$
\eta(X)=\eta_{0}+\frac{k_{\mathrm{B}} T}{32 \pi}\left(\frac{1}{D_{\eta}}+\frac{1}{2 D_{s}}\right) \log \frac{D_{\eta} k_{0}{ }^{2}}{|X|}+\cdots
$$

Here $\eta_{0}$ is the low-density value of the viscosity of hard disks, as predicted by the linear Boltzmann equation.

The result (66) agrees with the result quoted by Onuki. The normal stresses $P_{x x}$ and $P_{y y}$ are vanishing to the corresponding order in $X$.

For three-dimensional systems of hard spheres the shear stress is given by

$$
P_{x y}=-\eta(X) X=-\eta_{0} X-X|X|^{1 / 2} k_{\mathrm{B}} T\left(\frac{M_{x y}^{+-}}{\left(2 D_{s}\right)^{3 / 2}}+\frac{M_{x y}^{\eta \eta}}{\left(2 D_{\eta}\right)^{3 / 2}}\right)+\cdots
$$

and can be interpreted as a nonlinear viscosity $\eta(X)$, whereas $P_{x z}$ and $P_{y z}$ are vanishing to the corresponding order $|X|^{3 / 2}$. For the normal stresses we have obtained the result (62), similar to Eq. (67). The numerical values of the coefficients $M$ are listed in Table I. Here, $M^{+-}$represents the contributions for two opposite sound modes and $M^{\eta n}$ those of two shear modes. It should be stressed that the existence of normal stresses in steady shear flow in a dilute three-dimensional hard-sphere system shows that simple gases have already some very peculiar non-Newtonian fluid properties. ${ }^{(22)}$ It should perhaps also be mentioned that only differences of normal stresses, e.g., $\delta P_{x x}-\delta P_{y y}$, have direct physical significance.

2. Theoretical results for three-dimensional systems that are qualitatively the same as ours do exist already in the literature. Kawasaki and Gunton $(\mathrm{KG})^{(14)}$ and Yamada and Kawasaki $(\mathrm{YK})^{(15)}$ have evaluated the shear mode contributions to the shear and normal stresses in a fluid, using an extended mode coupling theory. Their results, expressed in coefficients $C_{i}$, are compared in Table II with our shear mode contributions $M^{m}$, using the relations $M_{x y}^{\eta n}=2 \sqrt{2} C_{0}, M_{x x}^{n n}=-2 \sqrt{2} C_{1}, M_{y y}^{n n}=-2 \sqrt{2} C_{2}$, and $M_{z z}^{n \eta}=-2 \sqrt{2} C_{3}$. Since the numerical values differ from ours, we have repeated the derivation of the singular part of the stress tensor for a general fluid using the extended 
Table II. Comparison with $M$ Values from the Literature in Units $10^{-2}$

\begin{tabular}{lllll}
\hline & $M_{x y}^{\eta \eta}$ & $M_{x x}^{\eta n}$ & $M_{y y}^{n \eta}$ & $M_{z z}^{n \eta}$ \\
\hline KG & -1.4 & +0.48 & -1.4 & +1.3 \\
YK & +0.86 & -3.0 & -12.0 & -1.6 \\
This work & -0.26 & +7.6 & -13.3 & +5.7 \\
\hline
\end{tabular}

mode coupling theory, and we obtained again Eq. (27), where $A_{x y}^{\lambda \mu}(\mathbf{k})$ are the amplitudes for general densities, defined in Ref. 8 as

$$
A_{x y}^{\lambda u}(\mathbf{k})=(1 / V)\left\langle a_{\mathbf{k}}^{\lambda *} a_{-\mathbf{k}}^{\mu *} J_{x y}\right\rangle_{\mathrm{eq}}
$$

and the matrix elements are given as

$$
\begin{aligned}
R_{v \rho}^{\lambda \mu}(k) & =\frac{1}{V}\left\langle a_{\mathbf{k}}^{\lambda *} a_{-\mathbf{k}}^{\mu *} \sum_{i=1}^{N}\left(x_{i} \frac{\partial}{\partial y_{i}}-v_{i x} \frac{\partial}{\partial v_{i y}}\right) a_{\mathbf{k}}{ }^{v} a_{-\mathbf{k}}^{o}\right\rangle_{\mathrm{eq}} \\
& \simeq \frac{1}{V}\left\langle a_{\mathbf{k}}^{\lambda *} a_{-\mathbf{k}}^{\mu *}\left(k_{y} \frac{d}{d k_{x}}-\sum_{i=1}^{N} v_{i x} \frac{\partial}{\partial v_{i y}}\right) a_{\mathbf{k}}{ }^{v} a_{-\mathbf{k}}^{o}\right\rangle_{\mathrm{eq}}
\end{aligned}
$$

Here $a_{\mathbf{k}}{ }^{\lambda}$ are the zeroth-order hydrodynamic modes, explicitly given in Ref. 8, and $\langle\cdots\rangle_{\text {eq }}$ is an average over an equilibrium ensemble. From this theory we recover again Eqs. (61) and (62) with exactly the same coefficients $M^{\eta n}$ for the shear mode contributions as given in Table I for the dilute gas of hard spheres. The contributions from the sound modes $\mathrm{M}^{+-}$have never been considered in the literature. They will in general depend explicitly on the density, and we have only given their low-density values in Table II.

3. We now remark on extension to higher densities and more complicated dynamical events. The theory developed here only takes into account the contributions from the Boltzmann equation and from the low-density ring events. We have incorporated neither excluded volume corrections to the Boltzmann equation and to the ring events, nor more complicated correlated collision sequences, such as rings within rings, etc. An extension to higher densities can be made along the lines followed by Dorfman and Cohen ${ }^{(23)}$ in describing the long-time tail of the velocity correlation function for fairly dense hard-sphere and hard-disk systems, since the long-time tails and the nonanalytic $X$ dependence of nonlinear transport coefficients are caused by the same mechanism. Another possibility is to extend the mode coupling theories, ${ }^{(14,15)}$ whose validity is independent of the density range considered.

More complicated dynamic events, especially ring-within-ring events, ${ }^{(10,24,25)}$ may be included into the kinetic theory. The analogy between the nonlinear transport coefficients obtained here and the wavenumber- 
dependent linear transport coefficients obtained by Pomeau ${ }^{(9)}$ and Ernst and Dorfman $^{(7,8)}$ suggests that in three dimensions these terms will give rise to a series of contributions proportional to $X^{P_{n}}$ with $P_{n}=1-2^{-n}$ and $n=$ $1,2, \ldots$. Including the ring within ring events is especially important in two dimensions, where their contributions for very small $X$ will be as important as those of the ring events themselves. A very similar situation occurs when discussing the time dependence of the generalized diffusion coefficient for hard disks. ${ }^{(24,25)}$ The analogy with this case suggests that a self-consistent formulation of the nonlinear kinetic theory, which is equivalent to a resummation of the terms $\sim(\log X)^{n}$, would lead to a nonlinear viscosity for hard disks proportional to $(\log X)^{1 / 2}$. All these problems are still under investigation.

4. The region of validity of the present theory is restricted by a number of requirements on the allowed values of $X$ :

(i) We first observe that the singular contributions to the stress tensor in Eq. (31) originate from $k$ values with $k_{m}<k<k_{0}$. The upper cutoff $k_{0}$ is determined by the relation $D_{n} k_{0}{ }^{2} \simeq v_{0} k_{0}$, where $v_{0} \equiv\left(k_{\mathrm{B}} T / m\right)^{1 / 2}$ is on the order of the thermal or sound velocity. In a fluid $k_{0} \simeq \sigma^{-1}$, i.e., on the order of the inverse range of the forces or the inverse hard-sphere $(d=3)$ or harddisk $(d=2)$ diameter; in gases $k_{0} \simeq n \sigma^{d-1}=n^{*} \sigma^{-1}$, i.e., on the order of the inverse mean free path. The lower cutoff is determined by the relation $D k_{m}^{2} \simeq X$, as can be seen from Eqs. (27) and (31); hence $X<D_{\eta} k_{0}^{2} \simeq v_{0} k_{0}$.

(ii) One finds a lower bound on $X$ by observing that our theory is only meaningful if the system is sufficiently large, i.e., if the lower cutoff $k_{m}>L^{-1}$, where $L$ is the linear dimension of the system. The condition is equivalent to $X L^{2} D_{n}^{-1} \simeq u L D_{n}^{-1}=\operatorname{Re}>1$, where $\operatorname{Re}$ is the Reynolds number. This condition is relevant to computer experiments (finite-size effects).

(iii) A reasonable theoretical criterion for $X$ is also that the change in flow velocity $\Delta u$ over the experimental region of size $L$ is small compared to the thermal velocity, i.e., $X=(\Delta u) L^{-1}<v_{0} L^{-1}$.

An alternative for an upper bound, which is, however, only available for three-dimensional systems, is obtained by requiring that the Reynolds number be smaller than the critical number, i.e., $X L^{2} D_{\eta}^{-1}<\mathrm{Re}^{\mathrm{er}} \approx 5000$. $^{(26)}$

(iv) The present theory is an asymptotic theory for small values of $X$, and hence the contributions to the nonlinear stress tensor should be ordered as $O(X)>O\left(X^{3 / 2}\right)>O\left(X^{2}\right)$ in three-dimensional systems, and $O(X)>$ $O(n X \ln X)>O\left(X^{2}\right)$ in two-dimensional systems, where also restrictions on the density are implied.

5. What are the orders of magnitude for the effects to be expected in typical experimental circumstances? The strongest condition (iii) yields $X<10^{4} \mathrm{sec}^{-1}$, using $L=1 \mathrm{~cm}, m=10^{-22} \mathrm{~g}$, and $T=300 \mathrm{~K}$. For air at STP $\left(\eta=2 \times 10^{-4} \mathrm{~g} \mathrm{~cm}^{-1} \mathrm{sec}^{-1}, D_{\eta}=\eta(\mathrm{nm})^{-1}=0.15 \mathrm{~cm}^{2} \mathrm{sec}^{-1}, \sigma \simeq\right.$ 
$10^{-8} \mathrm{~cm}, n \sigma^{3} \simeq 10^{-5}$, and $M \simeq 10^{-2}$ from Table I) a rough estimate gives, dropping numerical factors,

$$
\delta \eta / \eta \simeq|X|^{1 / 2} M k_{\mathrm{B}} T /\left(\eta D_{\eta}^{3 / 2}\right) \simeq|X|^{1 / 2} 10^{-10} \leqslant 10^{-8}
$$

and for water at STP $\left(\eta=10^{-2} \mathrm{~g} \mathrm{~cm}^{-1} \mathrm{sec}^{-1}, D_{\eta}=10^{-2} \mathrm{~cm}^{2} \mathrm{sec}^{-1}\right.$, and $M \simeq 10^{-2}$ ) this rough estimate gives

$$
\delta \eta / \eta \simeq|X|^{1 / 2} 10^{-11} \leqslant 10^{-9}
$$

The alternative condition (iii) involving Re $\mathrm{e}^{\mathrm{cr}}$ yields for air $X \simeq 10^{4} \mathrm{sec}^{-1}$ and for water $X \simeq 500 \mathrm{sec}^{-1}$, and we find essentially the same estimates. The effects in the normal stresses are one order of magnitude larger. In a typical rotating cylinder experiment $X \simeq 100 \mathrm{sec}^{-1}$. $^{(27)}$ Hence, the effects are extremely small in experimental circumstances.

Our theory, although restricted to low densities, gives a meaningful estimate for the magnitude of $\delta \eta / \eta$ in dense systems, since the coefficients $M^{\eta n}$ for the contributions of the shear modes are independent of the density, as can be seen from mode coupling theories (see remark 2).

What can and has been done in computer experiments? Recently a number of molecular dynamics experiments have been carried out by Naitoh and $\mathrm{Ono}^{(28)}$ and Ashurst and Hoover ${ }^{(29,30)}$ to determine the shear rate dependence of the shear viscosity. For these computer experiments on steadystate uniform shear flow in three-dimensional hard-sphere systems we read off the following data from graph $\mathrm{I}$ in Ref. 30: $X=2-7 \times 10^{-2} v_{0} \sigma^{-1}$, $\eta \simeq 7 m v_{0} \sigma^{-2}$, and $n^{*}=0.9$, so that $D_{\eta} \simeq 9 v_{0} \sigma$, and if our theory were applicable here, we would find for the shear mode contributions

$$
\frac{\delta \eta}{\eta}=\frac{M_{x y}^{\eta \eta}}{2 \sqrt{2}} \frac{v_{0}^{2} \sigma^{3}}{n^{*}} \frac{|X|^{1 / 2}}{D_{\eta}^{5 / 2}} \simeq 10^{-6}
$$

For densities around $n^{*}=0.3$ we obtain $D_{\eta}$ from the Enskog theory ${ }^{(17)}$ for a dense gas of hard spheres, using Ree and Hoover's ${ }^{(31)}$ values for Enskog's $\chi$ factor. The result is $D_{n} \simeq v_{0} \sigma$, and the predicted effect is about a factor 700 larger. This is still too small to be seen, given the uncertainty in the present computer experiments. The effects in the normal stresses are still a factor 30-50 larger than those in the shear stresses, as can be seen from Table II. However, our theory cannot be applied to the present computer experiments, since conditions (ii) and (iv) are violated. Condition (ii) is not fulfilled since the systems considered are too small. The linear dimension $L \simeq \sigma\left(N / n^{*}\right)^{1 / 3}$ of these systems with $N$ hard spheres $(N=108$ or 256$)$ at reduced densities $n^{*}=N \sigma^{3} / V$ around $n^{*}=0.9$ corresponds to $L \simeq 7 \sigma$, so that $\operatorname{Re}=X L^{2} D^{-1}$ $\simeq 0.1-0.4$, and finite-size effects dominate. At densities $n^{*} \simeq 0.3$ one has $L \simeq 10 \sigma$ and $\mathrm{Re} \simeq 2-7$, which are borderline cases where strong interference of finite-size effects and the effects of the hydrodynamic cutoff on the $k$ integral are to be expected. 
Next, the $X$ values considered by Naitoh and Ono are too large for our theory, although they satisfy the weaker bounds imposed by (i), i.e., $X \leqslant 0.1 D_{\eta} k_{0}^{2} \simeq 0.1 v_{0} \sigma^{-1}\left(k_{0} \simeq \sigma^{-1}, D_{\eta} \simeq v_{0} \sigma\right.$ for $\left.n^{*} \simeq 0.3\right)$ and by (iii), i.e., $X \leqslant v_{0} L^{-1} \simeq 0.1 v_{0} \sigma^{-1}$. The reason is that condition (iv) is violated, since the $O\left(X^{2}\right)$ terms in the pressure tensor are clearly dominating the terms of $O\left(X^{3 / 2}\right)$. This is so, since these investigators obtain consistent values for the nonlinear shear viscosity directly from momentum transport, as well as indirectly from the viscous heating of the fluid. The latter effect, which is of $O\left(X^{2}\right)$, should be negligibly small in our theory, as discussed in remark 8 . Considering the above restrictions in connection with the current computer experiments, one cannot expect to be able to verify the predictions of the present theory on hard-sphere systems.

In two-dimensional systems of hard disks we take the optimal density $n^{*}=0.3$, where $k_{0} \simeq \sigma^{-1}$ and $D_{\eta} \simeq 1.3 v_{0} \sigma$ (from Enskog's theory ${ }^{(17,31,32)}$ ), and find from Eq. (66)

$$
\delta \eta / \eta \simeq 0.02 \log \left(D_{\eta} k_{\mathrm{o}}^{2} /|X|\right)
$$

with

$$
L \sigma^{-1}<D_{n} k_{0}{ }^{2}|X|^{-1}<L^{2} \sigma^{-2}
$$

The inequalities (74) can be deduced from conditions (ii) and (iii) in remark 4. In typical computer experiments ${ }^{(1,2)}$ with a few thousand particles $L \simeq$ $\sigma\left(N / n^{*}\right)^{1 / 2} \simeq 100 \sigma$, so that the logarithm in Eq. (73) is between 5 and 10 , and $\delta \eta / \eta$ can be on the order of $10 \%$, which should be clearly visible. However, it remains to be checked whether $X$ can be made small enough (i.e., $L$ large enough) so that the viscous heating of the fluid can be neglected.

It would be extremely useful if computer experiments could give a check on the theory as developed so far for uniform shear flow, which seems feasible for systems of a few thousand hard disks.

6. The divergence of the viscosity for two-dimensional systems is connected to the contributions from small- $k$ or long-wavelength hydrodynamic modes of the ring collision operator. It is, of course, clear on physical grounds that the long-wavelength hydrodynamic modes should be affected by changes in the hydrodynamic fields on a scale of the wavelength of the modes. Here we have taken into account the effects of the slow, systematic change in the velocity fields on the long-wavelength hydrodynamic modes, and a convergent nonlinear expression for the pressure tensor results. The situation is not unlike that encountered in the continuum hydrodynamic theory of the flow of a fluid around a cylinder whose long axis is perpendicular to the direction of flow. There, the linear hydrodynamic equations must be modified by including the nonlinear Oseen terms ${ }^{(33)}$ before a well-behaved expression for 
the force exerted by the fluid on a cylinder can be obtained. In a kinetic description of this phenomenon the Oseen terms can be considered again as resulting from a delocalization of the Boltzmann operator. ${ }^{(34)}$ The relation between the force $\mathbf{F}$ exerted on the cylinder and the velocity $\mathbf{V}$ of the cylinder with respect to the fluid is similar to (59) and satisfies $\mathbf{V} \simeq \mathbf{F} \log |\mathbf{F}|$ for small F. Also here the difficulty with the linear theory is that it ignores the variations of the velocity field over large distances.

7. In this paper we have been using hydrodynamic modes for the unbounded system and neglected any hydrodynamic boundary conditions. If walls are present, interactions between walls and hydrodynamic modes give rise to interesting effects, which, although weak, are very long-ranged, due to the large extension in space of the hydrodynamic modes. ${ }^{(35)}$

8. We have neglected here the viscous heating of the fluid, which raises the temperature of the fluid during the time that the shear flow is taking place. However, as the rate of heating is proportional to $X^{2}$ for small $X$, it can be argued that we can consistently neglect the effects of the heating of the fluid. To see this one notes that, from the rough argument presented in Eq. (5), the time interval over which the pressure tensor develops to its asymptotic form is on the order of $|X|^{-1}$. Since the rate of heating is uniform, i.e., $\partial_{t} T=$ $\eta X^{2} / C_{V}$, the temperature change of the system over this time interval is proportional to $X^{2}|X|^{-1} \sim|X|$. For small $X$ this produces corrections to the pressure tensor that are of the order of $X^{2}$, and hence small compared to terms of order $|X| \log |X|$ or $|X|^{3 / 2}$. The same arguments apply to the differences of the normal stresses, $\delta P_{x x}-\delta P_{y y}$, etc.

9. A complete theory of hydrodynamic processes that takes into account gradients in density, temperature, and velocity, as well as time-dependent and nonlocal effects, has not yet been completed. Even for the case of steady-state heat conduction the theory is not yet understood.

10. Finally, we mention the special case of self-diffusion. It seems clear that the diffusion of a tagged particle in a fluid of mechanically identical particles maintained in equilibrium is an intrinsically linear process. Therefore, the equations describing self-diffusion under these circumstances are almost certainly linear, but nonlocal. However, it is interesting to consider the case of diffusion in a fluid in which there is also a shear flow. Here a phenomenon called Taylor diffusion will take place ${ }^{(36) .7}$; the density profile is modified by the shear flow and the variation of the velocity field over large distances must be taken into account. The structure of the diffusion equation becomes similar to Eqs. (37)-(38), and when the ring contribution to the diffusion coefficient is calculated this modified form of the diffusion equation must be used for the propagation of the diffusion mode.

${ }^{7}$ Dr. J. C. Lewis brought the similarity to Taylor diffusion to our attention and provided the references. 


\section{APPENDIX A}

We apply the procedure discussed in Section 2 to Eq. (11b), set $\partial_{t} g=0$, replace $h_{R}$ in Eq. (13) by $h_{\mathrm{B}}$, and neglect $g_{R}$, so that $g(12) \simeq \delta g(12)$. We can consider $\delta g(12)$ or $f(1) f(2) \delta g(12) \equiv F(12)$ as a function which depends on $\mathbf{v}_{1}, \mathbf{v}_{2}, \mathbf{r}_{12}$, and on $\mathbf{R}=\frac{1}{2}\left(\mathbf{r}_{1}+\mathbf{r}_{2}\right)$, or actually only on the $x$ component, $R_{x}$, in the case of uniform, steady shear flow with $u_{\alpha}(\mathbf{r})=\delta_{\alpha y}\left(u_{0}+x X\right)$. On the left-hand side of Eq. (11b) we are only interested in terms of $O(\delta)$ and $O(\delta X)$, and therefore, we expand ("delocalize") the function $f(3)$ in Eq. (11b) around the point $\mathbf{R}$. We further write $u_{y}(\mathbf{R})=u_{0}+R_{x} X \equiv u_{y}$, and introduce $u_{y}$ as a new variable instead of $R_{x}$, so that

$$
\left(\mathbf{v}_{1} \cdot \frac{\partial}{\partial \mathbf{r}_{1}}+\mathbf{v}_{2} \cdot \frac{\partial}{\partial \mathbf{r}_{2}}\right) F(12)=\mathbf{v}_{12} \cdot \frac{\partial}{\partial \mathbf{r}_{12}} F+\frac{1}{2} X\left(v_{1 x}+v_{2 x}\right) \frac{\partial}{\partial u_{y}} F
$$

Now $F\left(\mathbf{v}_{1} \mathbf{v}_{2} \mathbf{r}_{12} u_{y}\right)$ must be invariant under the combined translation and Galilei transformation $\left\{\mathbf{r}_{i} \rightarrow \mathbf{r}_{i}-x_{0} \hat{\mathbf{x}}-X x_{0} t \hat{\mathbf{y}} ; \mathbf{v}_{i} \rightarrow \mathbf{v}_{i}-X x_{0} \hat{\mathbf{y}}\right\}$, and hence, it can depend on $u_{y}$ only through the differences $\mathbf{v}_{i}-\mathbf{u}(\mathbf{R}) \equiv \mathbf{V}_{i}$. Equation (A1) can now be put into the form

$$
\begin{aligned}
\left(\mathbf{v}_{1} \cdot\right. & \left.\frac{\partial}{\partial \mathbf{r}_{1}}+\mathbf{v}_{2} \cdot \frac{\partial}{\partial \mathbf{r}_{2}}\right) F(12) \\
\quad & =\left[\mathbf{V}_{12} \cdot \frac{\partial}{\partial \mathbf{r}_{12}}-\frac{1}{2} X\left(V_{1 x}+V_{2 x}\right)\left(\frac{\partial}{\partial V_{1 y}}+\frac{\partial}{\partial V_{2 y}}\right)\right] F\left(\mathbf{V}_{1} \mathbf{V}_{2} \mathbf{r}_{12}\right)
\end{aligned}
$$

Next, we delocalize $f(3)$ in Eq. (11b), recalling that $\mathbf{r}_{3}=\mathbf{r}_{1}$ due to the presence of $\delta\left(\mathbf{r}_{13}\right)$ in $T(13)$, and we retain all terms up to $O(X)$, i.e.,

$$
f(3)=n \phi\left(\mathbf{V}_{3}\right)\left[1+\frac{1}{2} x_{12} X \beta m V_{3 y}+X h_{B}\left(\mathbf{V}_{3}\right)\right]
$$

Inserting (A2) and (A3) into Eq. (11b) yields

$$
\begin{aligned}
& {\left[\mathbf{V}_{12} \cdot \frac{\partial}{\partial \mathbf{r}_{12}}-\bar{\Lambda}\left(\mathbf{V}_{1}\right)-\bar{\Lambda}\left(\mathbf{V}_{2}\right)-T(12)-\frac{1}{2} X\left(V_{1 x}+V_{2 x}\right)\left(\frac{\partial}{\partial V_{1 y}}+\frac{\partial}{\partial V_{2 y}}\right)\right.} \\
& \left.\quad-\frac{1}{2} x_{12} X\left(1-P_{12}\right) \bar{\Lambda}_{R}\left(\mathbf{V}_{1} \mid \beta m V_{y}\right)-X\left(1+P_{12}\right) \bar{\Lambda}_{R}\left(\mathbf{V}_{1} \mid h_{\mathrm{B}}\right)\right] F\left(\mathbf{V}_{1} \mathbf{V}_{2} \mathbf{r}_{12}\right) \\
& \quad=n^{2} \phi\left(V_{1}\right) \phi\left(V_{2}\right) T(12)\left(1+P_{12}\right)\left[X h_{\mathrm{B}}\left(\mathbf{V}_{1}\right)+\delta h\left(\mathbf{V}_{1}\right)\right]
\end{aligned}
$$

and we have defined

$$
\begin{aligned}
\bar{\Lambda}\left(\mathbf{V}_{1}\right) & =n \int d \mathbf{V}_{2} T_{0}(12)\left(1+P_{12}\right) \phi\left(V_{2}\right) \\
\bar{\Lambda}_{R}\left(\mathbf{V}_{1} \mid h\right) & =n \int d \mathbf{V}_{2} T_{0}(12)\left(1+P_{12}\right) \phi\left(V_{2}\right) h\left(\mathbf{V}_{2}\right)
\end{aligned}
$$


Taking the Fourier transform of this equation and writing $\mathbf{v}_{i}$ instead of $\mathbf{V}_{i}$ gives us

$$
\begin{gathered}
{\left[i \mathbf{k} \cdot \mathbf{v}_{12}-\bar{\Lambda}\left(\mathbf{v}_{1}\right)-\bar{\Lambda}\left(\mathbf{v}_{2}\right)+X \bar{R}\left(\mathbf{v}_{1} \mathbf{v}_{2} \mathbf{k}\right)\right] F\left(\mathbf{v}_{1} \mathbf{v}_{2} \mathbf{k}\right)} \\
=n^{2} \phi\left(v_{1}\right) \phi\left(v_{2}\right) T_{0}(12)\left(1+P_{12}\right) h_{\mathrm{B}}\left(\mathbf{v}_{1}\right)
\end{gathered}
$$

with

$$
\begin{aligned}
\bar{R}\left(\mathbf{v}_{1} \mathbf{v}_{2} \mathbf{k}\right) \phi\left(v_{1}\right) \phi\left(v_{2}\right)= & \phi\left(v_{1}\right) \phi\left(v_{2}\right) R\left(\mathbf{v}_{1} \mathbf{v}_{2} \mathbf{k}\right) \\
= & -\frac{1}{2}\left(v_{1 x}+v_{2 x}\right)\left(\frac{\partial}{\partial v_{1 y}}+\frac{\partial}{\partial v_{2 y}}\right) \\
& +\frac{1}{2} \frac{d}{d i k_{x}}\left(1-P_{12}\right) \bar{\Lambda}_{R}\left(\mathbf{v}_{1} \mid \beta m v_{y}\right) \\
& -\left(1+P_{12}\right) \bar{\Lambda}_{R}\left(\mathbf{v}_{1} \mid h_{\mathrm{B}}\right)
\end{aligned}
$$

The first observation is that we have omitted two terms. This is allowed since we are only interested in the components $\int d \mathbf{v}_{1} d \mathbf{v}_{2} \psi^{\lambda}\left(\mathbf{v}_{1}\right) \psi^{\mu}\left(\mathbf{v}_{2}\right)$ [Eq. (A6)], where $\psi^{\lambda}$ and $\psi^{\mu}$ are summational invariants, and we have to show that the above components of the omitted terms cancel each other:

$$
\begin{aligned}
\iint d \mathbf{v}_{1} d \mathbf{v}_{2} \psi^{\lambda}\left(\mathbf{v}_{1}\right) \psi^{\mu}\left(\mathbf{v}_{2}\right) T_{0}(12)(2 \pi)^{-\alpha} \int d \mathbf{q} F\left(\mathbf{v}_{1} \mathbf{v}_{2} \mathbf{q}\right) \\
\quad \stackrel{?}{=}-n^{2} \iint d \mathbf{v}_{1} d \mathbf{v}_{2} \psi^{\lambda}\left(\mathbf{v}_{1}\right) \psi^{\mu}\left(\mathbf{v}_{2}\right) \phi\left(\mathbf{v}_{1}\right) \phi\left(\mathbf{v}_{2}\right) T_{0}(12)\left(1+P_{12}\right) \delta h\left(\mathbf{v}_{1}\right)
\end{aligned}
$$

On both sides of (A8) we may replace $\psi^{\lambda}\left(\mathbf{v}_{1}\right) \psi^{\mu}\left(\mathbf{v}_{2}\right)$ by $-\psi^{\lambda}\left(\mathbf{v}_{1}\right) \psi^{\mu}\left(\mathbf{v}_{1}\right)$, since the remaining part of the integral is symmetric under interchange of particles 1 and 2 , and since $\left[\psi^{\lambda}\left(\mathbf{v}_{1}\right)+\psi^{\lambda}\left(\mathbf{v}_{2}\right)\right]\left[\psi^{\mu}\left(\mathbf{v}_{1}\right)+\psi^{\mu}\left(\mathbf{v}_{2}\right)\right]$ is a left eigenfunction of $T_{0}(12)$ with zero eigenvalue. By writing

$$
F\left(\mathbf{v}_{1} \mathbf{v}_{2} \mathbf{k}\right)=n^{2} \phi\left(\mathbf{v}_{1}\right) \phi\left(\mathbf{v}_{2}\right) \delta G\left(\mathbf{v}_{1} \mathbf{v}_{2} \mathbf{k}\right)
$$

one sees that Eq. (A8) represents now the component of Eq. (17) along the products of hydrodynamic modes, and the above cancellation is justified.

In Section 3 we are especially interested in the following matrix elements of $R\left(\mathbf{v}_{1} \mathbf{v}_{2} \mathbf{k}\right)$ to $O\left(k^{0}\right)$ :

$$
\begin{aligned}
R_{v \rho}^{\lambda \mu}(\mathbf{k})=\iint & d \mathbf{v}_{1} d \mathbf{v}_{2} \chi_{\mathbf{k}}^{\lambda}\left(\mathbf{v}_{1}\right) \chi_{-\mathbf{k}}^{\mu}\left(\mathbf{v}_{2}\right) \bar{R}\left(\mathbf{v}_{1} \mathbf{v}_{2} \mathbf{k}\right) \\
& \times \chi_{\mathbf{k}}^{\nu}\left(\mathbf{v}_{1}\right) \chi_{-\mathbf{k}}^{\rho}\left(\mathbf{v}_{2}\right) \phi\left(v_{1}\right) \phi\left(v_{2}\right)
\end{aligned}
$$

where $\chi_{\mathbf{k}}{ }^{\lambda}(\mathbf{v})$ are the hydrodynamic modes of the linearized Boltzmann equation, i.e.,

$$
[i \mathbf{k} \cdot \mathbf{v}-\bar{\Lambda}(\mathbf{v})] \chi_{\mathbf{k}}{ }^{\lambda}(\mathbf{v}) \phi(v)=\phi(v)[i \mathbf{k} \cdot \mathbf{v}-\Lambda(\mathbf{v})] \chi_{\mathbf{k}}{ }^{\lambda}(\mathbf{v})=-z_{k}{ }^{\lambda} \chi_{\mathbf{k}}{ }^{\lambda}(\mathbf{v}) \phi(v)
$$


The modes are to $O\left(k^{0}\right)$ equal to the summational invariants $\psi_{\mathbf{k}}^{\lambda}(\mathbf{v})$ given in Eq. (25). The frequencies are given in Eq. (26) for small $k$, and the relevant mode pairs are such that

$$
z_{k}{ }^{\lambda}+z_{k}{ }^{\mu}=O\left(k^{2}\right)
$$

We will show below that for matrix elements between the relevant mode pairs the following relation holds, to $O\left(k^{0}\right)$ :

$$
\frac{1}{2} \frac{d}{d i k_{x}}\left[\bar{\Lambda}_{R}\left(\mathbf{v}_{1} \mid \beta m v_{y}\right)-\bar{\Lambda}_{R}\left(\mathbf{v}_{2} \mid \beta m v_{y}\right)\right]=-\frac{1}{2} v_{12 x}\left(\frac{\partial}{\partial v_{1 y}}-\frac{\partial}{\partial v_{2 y}}\right)-k_{y} \frac{d}{d k_{x}}
$$

As one can verify that the matrix elements of $\bar{\Lambda}_{R}(\mathbf{v} \mid h)$ in (A7) are $O(k)$, one can bring the operator $R$ in the following form, to $O\left(k^{0}\right)$ :

$$
\begin{aligned}
& \bar{R}\left(\mathbf{v}_{1} \mathbf{v}_{2} \mathbf{k}\right)=-k_{y} \frac{d}{d k_{x}}-v_{1 x} \frac{\partial}{\partial v_{1 y}}-v_{2 x} \frac{\partial}{\partial v_{2 y}} \\
& R\left(\mathbf{v}_{1} \mathbf{v}_{2} \mathbf{k}\right)=\bar{R}\left(\mathbf{v}_{1} \mathbf{v}_{2} \mathbf{k}\right)+\beta m v_{1 x} v_{1 y}+\beta m v_{2 x} v_{2 y}
\end{aligned}
$$

Proof of Eq. (A13): We first introduce the operator $S_{a}(i) f\left(\mathbf{v}_{i}\right) \equiv f\left(\mathbf{v}_{i}-a \hat{\mathbf{v}}_{y}\right)$, where $\hat{\mathbf{v}}_{y}$ is a unit vector along the $v_{y}$ axis, and we notice that

$$
\bar{\Lambda}_{R}\left(\mathbf{v}_{i} \mid \beta m v_{y}\right)=\lim _{a \rightarrow 0}\left[\frac{d}{d a} S_{a}(i), \bar{\Lambda}\left(\mathbf{v}_{i}\right)\right] \equiv\left[A(i), \bar{\Lambda}\left(\mathbf{v}_{i}\right)\right]
$$

where $[$,$] is the commutator. Introducing a short-hand notation for matrix$ elements of operators, defined in (A10) with the functions $\phi\left(\mathbf{v}_{1}\right)$ to the right of the operator, i.e.,

$$
R_{v \rho}^{\lambda \mu}(\mathbf{k})=\left(\lambda \mu\left|R\left(\mathbf{v}_{1} \mathbf{v}_{2} \mathbf{k}\right)\right| \nu \rho\right)
$$

then we can write for the left-hand side (lhs) of (A13), using (A15) and (A11),

$$
\begin{aligned}
&(\lambda \mu|\mathrm{h} s(\mathrm{~A} 13)| \nu \rho) \\
&=\frac{1}{2}\left(\lambda \mu\left|\frac{d}{d i k_{x}}\left\{\left[A(1), \bar{\Lambda}\left(\mathbf{v}_{1}\right)\right]-\left[A(2), \bar{\Lambda}\left(\mathbf{v}_{2}\right)\right]\right\}\right| v \rho\right) \\
&=\frac{1}{2}\left(\lambda \mu\left|\frac{d}{d i k_{x}}\left\{A(1)\left(i \mathbf{k} \cdot \mathbf{v}_{1}+z_{k}{ }^{\nu}\right)+A(2)\left(i \mathbf{k} \cdot \mathbf{v}_{2}-z_{k}{ }^{\rho}\right)\right\}\right| \nu \rho\right) \\
&-\frac{1}{2}\left(\lambda \mu\left|\left\{\left(i \mathbf{k} \cdot \mathbf{v}_{1}+z_{k}{ }^{\lambda}\right) A(1)+\left(i \mathbf{k} \cdot \mathbf{v}_{2}-z_{k}{ }^{\mu}\right) A(2)\right\} \frac{d}{d i k_{x}}\right| \nu \rho\right)
\end{aligned}
$$

Since we are interested in matrix elements of (A17) only to $O\left(k^{0}\right)$ and $O\left(k d / d k_{x}\right)$, and only between the mode pairs $\left(\lambda_{\mu}\right)$ and $(v \rho)$ satisfying Eq. 
(A12), we make everywhere the replacements $z_{k}{ }^{\nu} \rightleftharpoons-z_{k}{ }^{0}$ and $z_{k}{ }^{\lambda} \rightleftharpoons-z_{k}{ }^{\mu}$, and next we eliminate the frequencies, again using (A11). This yields

$$
\begin{aligned}
(\lambda \mu|\operatorname{lhs}(\mathrm{A} 13)| \nu \rho) & \\
= & \frac{1}{2}\left(\lambda \mu\left|\frac{d}{d i k_{x}}\left\{A(1)\left(i \mathbf{k} \cdot \mathbf{v}_{12}-\bar{\Lambda}\left(\mathbf{v}_{2}\right)\right)-A(2)\left(i \mathbf{k} \cdot \mathbf{v}_{12}-\bar{\Lambda}\left(\mathbf{v}_{1}\right)\right)\right\}\right| \nu \rho\right) \\
& -\frac{1}{2}\left(\lambda \mu\left|\left\{\left(i \mathbf{k} \cdot \mathbf{v}-\bar{\Lambda}\left(\mathbf{v}_{2}\right)\right) A(1)-\left(i \mathbf{k} \cdot \mathbf{v}_{12}-\bar{\Lambda}\left(\mathbf{v}_{1}\right)\right) A(2)\right\} \frac{d}{d i k_{x}}\right| \nu \rho\right) \\
= & (\lambda \mu|\operatorname{rhs}(\mathrm{A} 13)| \nu \rho)
\end{aligned}
$$

In obtaining the last equation we omitted the $\bar{\Lambda}$ terms, which are of $O\left(k^{2} d / d k_{x}\right)$, and we have used Eq. (A15).

In fact, there are many equivalent forms for $R\left(\mathbf{v}_{1} \mathbf{v}_{2} \mathbf{k}\right)$, which can be derived from (A10) in a less tricky way, e.g., one obtains in a relatively simple way

$$
\begin{aligned}
R\left(\mathbf{v}_{1} \mathbf{v}_{2} \mathbf{k}\right)= & -\frac{1}{2} \frac{d}{d k_{x}} \mathbf{k} \cdot \mathbf{v}_{12} v_{12 y} \beta m-\frac{1}{2}\left(v_{1 x}+v_{2 x}\right)\left(\frac{\partial}{\partial v_{1 y}}+\frac{\partial}{\partial v_{2 y}}\right) \\
& +\beta m v_{1 x} v_{1 y}+\beta m v_{2 x} v_{2 y}
\end{aligned}
$$

We prefer, however, the simple form (A11), which, in addition, shows the connection with the extended mode coupling theories.

\section{APPENDIX B}

In the three-dimensional case the solutions $B^{\lambda \mu}(\mathbf{k}, X)$ of the differential equations (37) and (41) contain still constant terms, $B^{\lambda \mu}(\mathbf{k}, 0)=$ $\beta A^{\lambda \mu}(\mathbf{k})\left(k^{2} D_{\lambda}+k^{2} D_{\mu}\right)^{-1}$. For the singular part of the stress tensor one only needs

$$
B^{\lambda \mu}(\mathbf{k}, X)-B^{\lambda \mu}(\mathbf{k}, 0) \equiv(1 /|X|) \delta B^{\lambda \mu}
$$

It is convenient to transform Eqs. (37) and (41) into differential equations for $\delta B\left(\mathbf{k}^{*}, X\right)$, and we introduce a dimensionless variable $\mathbf{k}^{*}$ by the relation

$$
2 D^{(n)} k^{2}=|X|\left(k^{*}\right)^{2}
$$

Its definition depends on the pair of modes involved, where $n=-1$ refers to $(+-) ; n=2$ to $\left(\eta_{1} \eta_{1}\right) ; n=1$ to $\left(\eta_{2} \eta_{1}\right)$; and $n=0$ to $\left(\eta_{2} \eta_{2}\right)$; and $D^{(n)}=D_{\text {s }}$ for $n=-1$ and $D^{(n)}=D_{n}$ for $n=2,1,0$. This yields the following set of equations, where $\theta=\operatorname{sg}\left(X k_{y}\right)$ and the asterisk is dropped again:

$$
\left(\frac{d}{d k_{x}}+\frac{n k_{x}}{k^{2}}-\frac{\theta k^{2}}{\left|k_{y}\right|}\right) \delta B^{(n)}(\mathbf{k}, X)=I^{(n)}(\mathbf{k})
$$


with boundary conditions

$$
\delta B^{(n)}\left(\mathbf{k}^{0}\left(2 D^{(n)} /|X|^{1 / 2}\right), X\right)=0
$$

as follows from (43) and (B1). The functions $I^{(n)}$ are found to be

$$
\begin{aligned}
I^{(-1)}(\mathbf{k}) & =-k_{y}\left(k^{2}-5 k_{x}^{2}\right) / k^{6} \\
I^{(2)}(\mathbf{k}) & =2 k_{y}\left(k^{2}-2 k_{x}{ }^{2}\right) / k^{6} \\
I^{(1)}(\mathbf{k}) & =k_{z} \delta B^{(2)}(\mathbf{k}, X) / k_{y} k \\
I^{(0)}(\mathbf{k}) & =-2 k_{z}\left[k^{-3} k_{z}+\delta B^{(1)}(\mathbf{k}, X)\right] / k_{y} k
\end{aligned}
$$

The solution of (B3a) that satisfies the boundary condition (B3b) is

$$
\delta B^{(n)}(\mathbf{k}, X)=-\int_{k_{x}}^{\theta \kappa_{0}} d k_{x}{ }^{\prime}\left(k^{\prime} / k\right)^{n} K\left(\theta \mathbf{k}, \theta \mathbf{k}^{\prime}\right) I^{(n)}\left(\mathbf{k}^{\prime}\right)
$$

with $\kappa_{0}=k_{0}\left(2 D^{(n)} /|X|\right)^{1 / 2}$ and

$$
K\left(\mathbf{k}, \mathbf{k}^{\prime}\right)=\exp \left\{\left|k_{y}\right|^{-1}\left[k_{x}\left(k^{2}-\frac{2}{3} k_{x}^{2}\right)-k_{x}{ }^{\prime}\left(k^{\prime 2}-\frac{2}{3} k_{x}^{\prime 2}\right)\right]\right\}
$$

From Eqs. (31)-(34) and (B1) we find the singular part of the pressure tensor as

$$
\delta P_{\alpha \beta}=-X|X|^{1 / 2} \beta^{-1} \sum_{n=-1,0,1,2} M_{\alpha \beta}^{(n)}\left(2 D^{(n)}\right)^{-3 / 2}
$$

where $M_{\alpha \beta}^{(n)}$ is the limiting behavior for $X \rightarrow 0$ or $\kappa_{0} \rightarrow \infty$ of the form

$$
\begin{aligned}
M_{\alpha \beta}^{(n)}\left(\kappa_{0}\right) & =(2 \pi)^{-3} \int^{\prime} d \mathbf{k} A_{\alpha \beta}^{(n)}(\mathbf{k}) \delta B^{(n)}(\mathbf{k}) \\
& =-2(2 \pi)^{-3} \int^{\prime \prime} d \mathbf{k} \int_{k_{x}}^{\kappa_{0}} d \mathbf{k}_{x}\left(k^{\prime} / k\right)^{n} K\left(\mathbf{k}, \mathbf{k}^{\prime}\right) A_{\alpha \beta}^{(n)}(\mathbf{k}) I^{\langle n)}\left(\mathbf{k}^{\prime}\right)
\end{aligned}
$$

The prime on the integral sign in (B8a) indicates that $k<\kappa_{0}$; the double prime on the integral sign in (B8b) indicates that $k<\kappa_{0}$ and that we have restricted $k_{y}$ to the interval $X k_{y}>0$ by means of Eq. (53), which can be deduced in the three-dimensional case from (B4), (B5), (34), and

$$
\beta A_{\alpha \beta}^{+-}=A_{\alpha \beta}^{(-1)}, \quad \beta A_{\alpha \beta}^{\eta_{2} \eta_{1}}=A_{\alpha \beta}^{(1)}, \quad \frac{1}{2} \beta A_{\alpha \beta}^{\eta_{1} \eta_{1}}=A_{\alpha \beta}^{(2)}, \quad \frac{1}{2} \beta A_{\alpha \beta}^{\eta_{2} \eta_{2}}=A_{\alpha \beta}^{(0)}
$$

The next observation is that the integrand in (B8) is an odd function of $k_{z}$ for $(\alpha \beta)=(y z)$ or $(z x)$, as can be verified from (34), (40), and (B4) (B6). Hence we find

$$
\delta P_{y z}=\delta P_{z x}=0
$$

Consider first the contributions in (B8) from the sound modes $(n=-1)$. 
We insert (B4) into (B8), restrict $k_{z}$ to be $>0$, which yields a factor 2 , and introduce new variables

$$
k_{y}=q \cos \phi, \quad k_{z}=q \sin \phi \quad \text { or } \quad \mathbf{q}=\left(k_{y}, k_{z}\right)
$$

with

$$
\begin{array}{lll}
\phi \in\left[0, \frac{1}{2} \pi\right] & \text { if } & \operatorname{sg}(X)=1 \\
\phi \in\left[\frac{1}{2} \pi, \pi\right] & \text { if } & \operatorname{sg}(X)=-1
\end{array}
$$

The integration region over $\left(k_{x}, k_{x}{ }^{\prime}\right)$ is divided into regions $\mathbf{I}$ and II, defined as

$$
\begin{gathered}
\{I\}=\left\{0<k_{x}{ }^{\prime}<\kappa_{0} ;-k_{x}{ }^{\prime}<k_{x}<k_{x}{ }^{\prime}\right\} \\
\{I I\}=\left\{-\kappa_{0}<k_{x}<0 ; k_{x}<k_{x}{ }^{\prime}<-k_{x}\right\}
\end{gathered}
$$

In region I we introduce

$$
k_{x}^{\prime}=q x ; \quad k_{x}=k_{x}^{\prime} y=q x y
$$

and in region II

$$
k_{x}=-q x ; \quad k_{x}^{\prime}=k_{x} y=-q x y
$$

with

$$
x \in\left[0, \kappa_{0} / q\right] ; \quad y \in[-1,1]
$$

Since we are only interested in the dominant small- $X$ behavior, we take the limit $X \rightarrow 0$ or $\kappa_{0} \rightarrow \infty$ everywhere. This yields for the contribution to $M_{\alpha \beta}^{(-1)}$ from region $\mathrm{I}$

$$
\begin{aligned}
M_{\alpha \beta}^{(-1)}(I)= & 4(2 \pi)^{-3} \int_{0}^{\infty} d x \int_{-1}^{1} d y \int^{\prime \prime \prime} d \phi \int_{0}^{\infty} d q \\
& \times x \cos \phi \exp \left(-q^{2} x E \||\cos \phi|\right) \\
& \times\left[A_{\alpha \beta}^{(-1)}(q x y, \mathbf{q})\left(1-4 x^{2}\right)\left(1+x^{2} y^{2}\right)^{1 / 2}\left(1+x^{2}\right)^{-7 / 2}\right]
\end{aligned}
$$

The triple prime refers to the restrictions in (B11b). The contribution from region II follows similarly, and its explicit form can be obtained from (B14) by replacing inside the square brackets $x y$ by $-x$, and $x$ by $-x y$. We have further written

$$
K\left(\mathbf{k}, \mathbf{k}^{\prime}\right)=\exp \left(-q^{2} x E /|\cos \phi|\right)
$$

with

$$
E=1-y+\frac{1}{3} x^{2}\left(1-y^{3}\right)
$$


and observe that the exponential function in (B14) is the only $q$-dependent factor, as can be seen from (B4), (B9), and (51). Hence

$$
\int_{0}^{\infty} d q \exp \left(-q^{2} x E /|\cos \phi|\right)=\frac{1}{2}(\pi|\cos \phi| \mid x E)^{1 / 2}
$$

Now we use the explicit expressions (34) for the amplitudes and integrate over the angle $\phi$, using

$$
\int_{0}^{\pi / 2} d \phi(\sin \phi)^{r}(\cos \phi)^{s}=\frac{1}{2} \Gamma\left(\frac{r+1}{2}\right) \Gamma\left(\frac{s+1}{2}\right) / \Gamma\left(\frac{r+s+2}{2}\right)
$$

The result is

$$
\begin{gathered}
M_{x y}^{(-1)}=\left(\gamma / 10 \pi^{2}\right) N(1) \\
\operatorname{sg}(X)\left[\begin{array}{l}
M_{x x}^{(-1)} \\
M_{y y}^{(-1)} \\
M_{z z}^{(-1)}
\end{array}\right]=\left(8 \pi^{2} \gamma\right)^{-1}\left[\begin{array}{l}
-1 \\
5 / 7 \\
2 / 7
\end{array}\right] N(2)+\left(8 \pi^{2} \gamma\right)^{-1}\left[\begin{array}{r}
2 / 3 \\
-1 / 3 \\
-1 / 3
\end{array}\right] N(3)
\end{gathered}
$$

with the following abbreviations:

$$
\gamma=\Gamma(7 / 4) / \Gamma(5 / 4)=1.0140
$$

and

$$
N(1)=\int_{0}^{\infty} d x \int_{-1}^{1} d y\left(\frac{x}{E}\right)^{1 / 2}\left[\frac{x y\left(1-4 x^{2}\right)}{\left(1+x^{2} y^{2}\right)^{1 / 2}\left(1+x^{2}\right)^{7 / 2}}-\frac{x\left(1-4 x^{2} y^{2}\right)}{\left(1+x^{2}\right)^{1 / 2}\left(1+x^{2} y^{2}\right)^{7 / 2}}\right]
$$

$N(2)=\int_{0}^{\infty} d x \int_{-1}^{1} d y\left(\frac{x}{E}\right)^{1 / 2}\left[\frac{1-4 x^{2}}{\left(1+x^{2} y^{2}\right)^{1 / 2}\left(1+x^{2}\right)^{7 / 2}}+\frac{1-4 x^{2} y^{2}}{\left(1+x^{2}\right)^{1 / 2}\left(1+x^{2} y^{2}\right)^{7 / 2}}\right]$

$$
N(3)=\int_{0}^{\infty} d x \int_{-1}^{1} d y\left(\frac{x}{E}\right)^{1 / 2}\left[\frac{\left(1+x^{2} y^{2}\right)^{1 / 2}\left(1-4 x^{2}\right)}{\left(1+x^{2}\right)^{7 / 2}}+\frac{\left(1+x^{2}\right)^{1 / 2}\left(1-4 x^{2} y^{2}\right)}{\left(1+x^{2} y^{2}\right)^{7 / 2}}\right]
$$

Next, we turn to the shear mode contributions. The calculation of the $\left(\eta_{1} \eta_{1}\right)$-mode contribution is completely analogous. The result is

$$
\begin{aligned}
M_{x y}^{(2)} & =\left(\gamma / 5 \pi^{2}\right) N(4) \\
\operatorname{sg}(X)\left[\begin{array}{l}
M_{x x}^{(2)} \\
M_{y y}^{(2)} \\
M_{z z}^{(2)}
\end{array}\right] & =\left(4 \pi^{2} \gamma\right)^{-1}\left[\begin{array}{l}
-1 \\
5 / 7 \\
2 / 7
\end{array}\right] N(5)+\left(6 \pi^{2} \gamma\right)^{-1}\left[\begin{array}{r}
1 \\
-8 / 7 \\
1 / 7
\end{array}\right] N(6)
\end{aligned}
$$


with

$$
\begin{aligned}
& N(4)=\int_{0}^{\infty} d x \int_{-1}^{1} d y\left(\frac{x}{E}\right)^{1 / 2} \frac{\left(1-x^{2}\right) x y-x\left(1-x^{2} y^{2}\right)}{\left(1+x^{2} y^{2}\right)^{2}\left(1+x^{2}\right)^{2}} \\
& N(5)=\int_{0}^{\infty} d x \int_{-1}^{1} d y\left(\frac{x}{E}\right)^{1 / 2} \frac{2-x^{2}-x^{2} y^{2}}{\left(1+x^{2} y^{2}\right)^{2}\left(1+x^{2}\right)^{2}} \\
& N(6)=\int_{0}^{\infty} d x \int_{-1}^{1} d y\left(\frac{x}{E}\right)^{1 / 2} \frac{1-x^{4} y^{2}}{\left(1+x^{2} y^{2}\right)^{2}\left(1+x^{2}\right)^{2}}
\end{aligned}
$$

where $E$ is given in (B15b). To obtain the contribution $M_{\alpha \beta}^{(1)}$ from the $\left(\eta_{2} \eta_{1}\right)$ modes we start again from (B8), use (B4), and insert (B5) for $\delta B^{(2)}$, using again (B4). Since $K\left(\mathbf{k}, \mathbf{k}^{\prime}\right) K\left(\mathbf{k}^{\prime}, \mathbf{k}^{\prime \prime}\right)=K\left(\mathbf{k}, \mathbf{k}^{\prime \prime}\right)$, we write

$$
\begin{aligned}
M_{\alpha \beta}^{(1)}\left(\kappa_{0}\right)= & 4(2 \pi)^{-3} \int^{\prime \prime} d \mathbf{k} \int_{k_{x}}^{\kappa_{0}} d k_{x}^{\prime \prime} \int_{k_{x}}^{k_{x}^{\prime \prime}} d k_{x}{ }^{\prime}\left(k^{\prime}\right)^{-2} K\left(\mathbf{k}, \mathbf{k}^{\prime \prime}\right) \\
& \times\left(k_{z} / k\right)\left(k^{\prime \prime}\right)^{-4}\left(q^{2}-k_{x}^{\prime \prime 2}\right) A_{\alpha \beta}^{(1)}(\mathbf{k})
\end{aligned}
$$

and carry out the $k_{x}^{\prime}$ integration

$$
\int_{k_{x}}^{k_{x}^{\prime \prime}} d k_{x}^{\prime}\left(k^{\prime}\right)^{-2}=q^{-1} A\left(k_{x}^{\prime \prime}, k_{x}\right)
$$

with

$$
A\left(k_{x}^{\prime \prime}, k_{x}\right)=\tan ^{-1}\left(k_{x}^{\prime \prime} / q\right)-\tan ^{-1}\left(k_{x} / q\right)
$$

From here on everything proceeds as before, and we arrive at

$$
\begin{aligned}
& M_{x y}^{(1)}=\left(4 \gamma / 15 \pi^{2}\right) N(7) \\
& \operatorname{sg}(X)\left[\begin{array}{l}
M_{x x}^{(1)} \\
M_{y y}^{(1)} \\
M_{z z}^{(1)}
\end{array}\right]=\left(7 \pi^{2} \gamma\right)^{-1}\left[\begin{array}{r}
0 \\
-1 \\
1
\end{array}\right] N(8)
\end{aligned}
$$

In the case of the $\left(\eta_{2} \eta_{2}\right)$ modes $(n=0)$, we see from (B10), (34), and (40) that $A_{x y}^{(0)}=0$, so that

$$
M_{x y}^{(0)}=0
$$

In the coefficients $M_{a a}^{(0)}$ for the normal stresses we can perform two intermediate $k_{x}$ integrations, with the final result

$$
\operatorname{sg}(X)\left[\begin{array}{l}
M_{x x}^{(0)} \\
M_{y y}^{(0)} \\
M_{z z}^{(0)}
\end{array}\right]=\left(6 \pi^{2} \gamma\right)^{-1}\left[\begin{array}{r}
1 \\
-11 / 7 \\
4 / 7
\end{array}\right] N(9)
$$


The symbols $N(i)$ with $i=7,8,9$ stand for

$$
\begin{aligned}
& N(7)=\int_{0}^{\infty} d x \int_{-1}^{1} d y\left(\frac{x}{E}\right)^{1 / 2} A \frac{1-x^{4} y^{2}}{\left(1+x^{2}\right)^{2}\left(1+x^{2} y^{2}\right)^{2}} \\
& N(8)=\int_{0}^{\infty} d x \int_{-1}^{1} d y\left(\frac{x}{E}\right)^{1 / 2} A\left[\frac{x y\left(1-x^{2}\right)}{\left(1+x^{2} y^{2}\right)\left(1+x^{2}\right)^{2}}-\frac{x\left(1-x^{2} y^{2}\right)}{\left(1+x^{2}\right)\left(1+x^{2} y^{2}\right)^{2}}\right] \\
& N(9)=\int_{0}^{\infty} d x \int_{-1}^{1} d y\left(\frac{x}{E}\right)^{1 / 2}\left[\frac{1+A^{2}\left(1-x^{2}\right)}{\left(1+x^{2}\right)^{2}}+\frac{1+A^{2}\left(1-x^{2} y^{2}\right)}{\left(1+x^{2} y^{2}\right)^{2}}\right]
\end{aligned}
$$

with

$$
A=\tan ^{-1} x-\tan ^{-1} x y
$$

The above integrals can be evaluated numerically directly, except $N(3)$ in the representation (B21c). The reason is that the contributions from each term in binomial $1-4 x^{2} y^{2}$ separately diverge for large $x$ and small $y$. The $x$ integration converges only after the $y$ integral is performed, which makes the representation (B21c) not suitable for numerical integration. Therefore, we derive a different expression for $N(3)$, i.e., the sound mode contribution to the normal stresses $\delta P_{a a}^{(+-)}$.

We start from Eq. (37) and write $B^{+-}(\mathbf{k}, X) \equiv B^{\langle-1\rangle}\left(\mathbf{k}^{*}, X\right)$ with $2 D_{\mathrm{s}} k^{2} \equiv|X|\left(k^{*}\right)^{2}$; then Eq. (37) takes the form (B3a) with the replacements (dropping the asterisk in $\mathbf{k}^{*}$ ):

$$
\delta B^{(-1)}(\mathbf{k}, X) \rightarrow B^{(-1)}(\mathbf{k}, X), \quad I^{(-1)}(\mathbf{k}) \rightarrow-\frac{1}{X} \frac{k_{x}}{k^{2}}
$$

The boundary condition is the same as in Eq. (47a). The solution is similar to Eq. (48), and the term depending on the boundary value gives a vanishing contribution to the normal stresses $\delta P_{a a}^{(+-)}$. Following the steps leading to (B8b), we obtain the alternative expression

$$
\operatorname{sg}(X) M_{a a}^{(-1)}=-\frac{4}{(2 \pi)^{3}} \int^{\prime \prime} d \mathbf{k} \int_{k_{x}}^{\infty} d k_{x}{ }^{\prime}\left(\frac{k_{a}^{\prime 2}}{k^{\prime 2}}-\frac{1}{3}\right) \frac{k^{\prime}}{k} K\left(\mathbf{k}, \mathbf{k}^{\prime}\right) \frac{k_{x}{ }^{2}}{k^{2}}
$$

The double prime on the $\mathbf{k}$ integral indicates that $k_{y}>0$ and $k_{z}>0$. In order to obtain (B35) we have interchanged the order of integrations over $k_{x}$ and $k_{x}{ }^{\prime}$, and relabeled $k_{x} \rightleftharpoons-k_{x}{ }^{\prime}$, using $K\left(\mathbf{k}, \mathbf{k}^{\prime}\right)=K\left(-\mathbf{k}^{\prime},-\mathbf{k}\right)$. We now change to new variables $\mathbf{k}=k \hat{\mathbf{k}}$ with $\hat{k}_{x}=\cos \theta, \hat{k}_{y}=\sin \theta \cos \phi$, and $\hat{k}_{z}=$ $\sin \theta \sin \phi$, where $0<\phi<\frac{1}{2} \pi$ and $0<\theta<\pi$. We then set $k_{x}{ }^{\prime}=k\left(\hat{k}_{x}+s\right)$, so that $k^{\prime} k^{-1} \equiv L\left(\hat{k}_{x}, s\right)=\left(1+2 s \hat{k}_{x}+s^{2}\right)^{1 / 2}$ and we carry out the $k$ integration, yielding

$$
\int_{0}^{\infty} d k k^{2} K\left(\mathbf{k}, \mathbf{k}^{\prime}\right)=\frac{1}{4} \sqrt{\pi} F\left(\hat{k}_{x}, s\right)\left(\hat{k}_{y} / s\right)^{3 / 2}
$$


Table III

\begin{tabular}{llllll}
\hline$i$ & $N(i)$ & $i$ & $N(i)$ & $i$ & $N(i)$ \\
\hline 1 & -0.395 & 4 & -0.373 & 7 & +0.185 \\
2 & +0.458 & 5 & +1.201 & 8 & -0.486 \\
3 & +0.292 & 6 & +0.727 & 9 & +5.63 \\
\hline
\end{tabular}

with

$$
F\left(\hat{k}_{x}, s\right)=\left(1+s \hat{k}_{x}+\frac{1}{3} s^{2}\right)^{-3 / 2}
$$

Finally we perform the $\phi$ integration using (B17), and we deduce from definition (B19),

$$
\begin{aligned}
N(3) & =-\frac{1}{2} \int_{0}^{\infty} d s \int_{0}^{\pi} d \theta(\sin \theta)^{5 / 2}(\cos \theta) F(\cos \theta, s) L(\cos \theta, s) s^{-3 / 2} \\
& =\int_{0}^{\infty} d y \int_{0}^{1} d x\left(1-x^{2}\right)^{3 / 4} x y^{-2}\left\{F_{-} L_{-}-F_{+} L_{+}\right\}
\end{aligned}
$$

Here $F_{ \pm}=F\left( \pm x, y^{2}\right)$ and $L_{ \pm}=L\left( \pm x, y^{2}\right)$. The difference inside braces contains $y^{2}$ as a factor, so that the integrand of (B38b) is free from singularities, and lends itself to direct numerical integration. Of course, we could write an analogous expression for $N(2)$, but we refrain from doing so since (B21b) is already in a suitable form.

The above method, with substitutions analogous either to (B34) or to (B1) and (B4), with or without relabelings $k_{x} \rightleftharpoons-k_{x}{ }^{\prime}$, generates different representations for the integrals $N(i)$, several of which have been used here as checks on the numerical results. However, the convergence of the representations for $N(3)$ is always rather subtle, and only expression (B38b) seemed suitable for numerical integration. The numerical values of $N(i)$ are given in Table III. In the body of the paper the factors $\operatorname{sg}(X)$ on the right-hand side of (B19), (B23), (B29), (B31), and (B35) are absorbed in the symbols $M_{a a}^{(n)}$.

\section{ACKNOWLEDGMENTS}

It is a pleasure to thank Dr. J. C. Lewis and Mr. C. Stolk for evaluating the integrals in Appendix B, and Prof. R. Zwanzig, M. Bixon, and J. Dufty for making helpful comments. One of us (MHE) thanks the Institute for Physical Science and Technology for the opportunity to spend the summer of 
1976 at the University of Maryland, where this research was started, and one of us (BC) wants to express his gratitude for the opportunity to spend the spring semester of 1977 at the University of Utrecht.

\section{REFERENCES}

1. B. J. Alder and T. E. Wainwright, Phys. Rev. A 1:18 (1970).

2. W. W. Wood, in Fundamental Problems in Statistical Mechanics III, E. G. D. Cohen, ed. (North-Holland, Amsterdam, 1975).

3. J. R. Dorfman and E. G. D. Cohen, Phys. Rev, A 6:776 (1972).

4. M. H. Ernst, E. H. Hauge, and J. M. J. van Leeuwen, Phys. Rev. A 4:2055 (1971).

5. Y. Pomeau and P. Resibois, Phys. Rev. Lett. C. (Netherlands) 19C:63 (1975) and references therein.

6. R. Zwanzig, Ann. Rev. Phys. Chem. 16:67 (1965).

7. M. H. Ernst and J. R. Dorfman, Physica 61:157 (1972).

8. M. H. Ernst and J. R. Dorfman, J. Stat. Phys. 12:311 (1975).

9. Y. Pomeau, Phys. Rev. A 5:2569 (1972); 7:1134 (1973); Phys. Lett. 38A:245 (1972).

10. I. de Schepper and M. H. Ernst, Generalized Hydrodynamics for the Diffusion Process, PhD diss., Univ. of Nijmegen (1975).

11. J. Keyes and I. Oppenheim, Physica 70:100 (1973).

12. J. Dufty and J. A. McLennan, Phys. Rev. 9:1266 (1974).

13. J. Sharma, PhD diss., Univ. of Maryland (1977).

14. K. Kawasaki and J. D. Gunton, Phys. Rev. A 8:2048 (1973).

15. T. Yamada and K. Kawasaki, Prog. Theor. Phys. 53:111 (1975).

16. A. Onuki, Transport Coefficients of Dense Fluids and Long Range Correlations in Non-Equilibrium Gases, PhD diss., Univ. of Tokyo (1975).

17. S. Chapman and T. G. Cowling, The Mathematical Theory of Non-uniform Gases (Cambridge at the University Press, 1960).

18. J. H. Ferziger and H. G. Kaper, Mathematical Theory of Transport Processes in Gases (North-Holland, Amsterdam, 1972).

19. M. H. Ernst, J. R. Dorfman, W. R. Hoegy, and J. M. J. van Leeuwen, Physica 45:127 (1969).

20. E. G. D. Cohen, Statistical Mechanics at the Turn of the Decade, E. G. D. Cohen, ed. (Marcel Dekker, New York, 1971), p. 49.

21. P. Resibois, Y. Pomeau, and J. Piasecki, J. Math. Phys. 15:1238 (1974).

22. R. B. Bird, Ann. Rev. Fluid Mech. 8:13 (1976).

23. J. R. Dorfman and E. G. D. Cohen, Phys. Rev. A 12:292 (1975).

24. I. M. de Schepper, H. Van Beijeren, and M. H. Ernst, Physica 75:1 (1974).

25. I. M. de Schepper and M. H. Ernst, Physica 87A:35 (1977).

26. A. A. Townsend, in Handbook of Fluid Dynamics, V. L. Streeter, ed. (McGraw-Hill, New York, 1961).

27. S. Chandrasekhar, Hydrodynamic and Hydromagnetic Stability (Oxford Univ. Press, 1961).

28. T. Naitoh and S. Ono, Phys. Lett. A57:448 (1976).

29. W. T. Ashurst and W. G. Hoover, Phys. Rev. A 11:658 (1975); W. G. Hoover and W. T. Ashurst, in Theoretical Chemistry, Advances and Perspectives, H. Eyring and D. Henderson, eds. (Academic Press, 1975), Vol. 1, p. 1.

30. W. T. Ashurst and W. G. Hoover, Phys. Lett. 61A:175 (1977).

31. F. H. Ree and W. G. Hoover, J. Chem. Phys. 40:939 (1964). 
270 M. H. Ernst, B. Cichocki, J. R. Dorfman, J. Sharma, and H. van Beijeren

32. D. M. Gass, J. Chem. Phys. 54:1898 (1971).

33. H. Lamb, Hydrodynamics (Dover, New York, 1945); L. D. Landau and E. M. Lifshitz, Fluid Mechanics (Pergamon Press, London, 1959).

34. J. R. Dorfman, H. Van Beijeren, and C. F. McClure, Archiwum Mechaniki Stosowanej 28: 333 (1976); and to be published.

35. P. Wolynes, Phys. Rev. A 13:1235 (1976).

36. G. Taylor, Proc. Roy. Soc. Lond. A 219:186 (1953); R. Aris, Proc. Roy. Soc. Lond. A 235:67 (1956). 